\title{
Conf $-940738--12$
}

UCRL-JC-119798

PREPRINT

\section{Criteria for Calculating the Efficiency of Deep-Pleated HEPA Filters with Aluminum Separators During and After Design Basis Accidents}

\author{
W. Bergman \\ M.W. First \\ W.L. Anderson \\ H. Gilbert \\ J.W. Jacox \\ This paper was prepared for submittal to the \\ 23rd DOEINRC Nuclear Air Cleaning and Treatment Conference \\ Buffalo, NY \\ July 24-28, 1994
}

February 1995

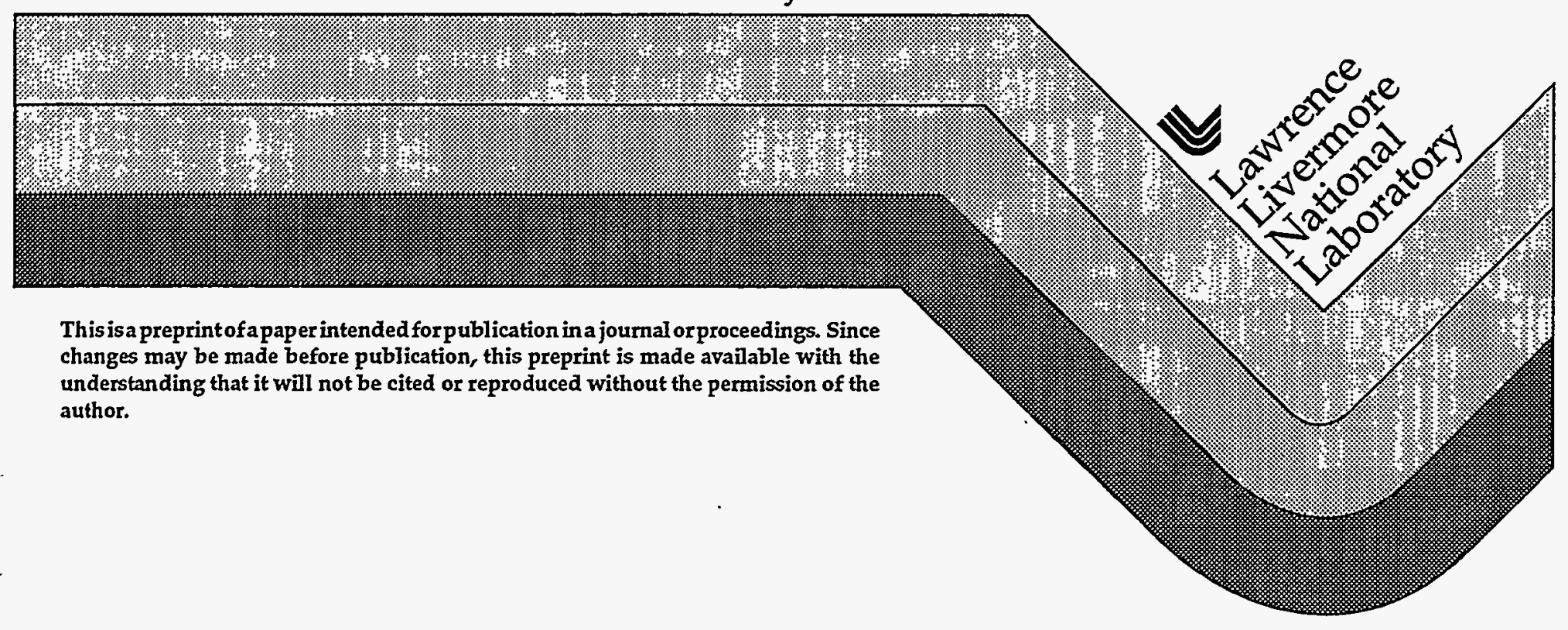




\section{DISCLAIMER}

This document was prepared as an account of work sponsored by an agency of the United States Government. Neither the United States Government nor the University of California nor any of their employees, makes any warranty, express or implied, or assumes any legal liability or responsibility for the accuracy, completeness, or usefulness of any information, apparatus, product, or process disclosed, or represents that its use would not infringe privately owned rights. Reference herein to any specific commercial product, process, or service by trade name, trademark, manufacturer, or otherwise, does not necessarily constitute or imply its endorsement, recommendation, or favoring by the United States Government or the University of California. The views and opinions of authors expressed herein do not necessarily state or reflect those of the United States Government or the University of California, and shall not be used for advertising or product endorsement purposes. 


\section{DISCLAIMER}

Portions of this document may be illegible in electronic image products. Images are produced from the best available original document. 


\title{
23rd DOE/NRC NUCLEAR AIR CLEANING AND TREATMENT CONFERENCE
}

\author{
CRITERIA FOR CALCULATING THE EFFICIENCY OF DEEP-PLEATED HEPA \\ FILTERS WITH ALUMINUM SEPARATORS DURING AND AFTER DESIGN BASIS ACCIDENTS* \\ W. Bergman, M. W. First ${ }^{1}$, W. L. Anderson 2 , H. Gilbert 3 , and J. W. Jacox 4 \\ Lawrence Livermore National Laboratory \\ P.O. Box 5505, Livermore, CA 94550
}

\begin{abstract}
We have reviewed the literature on the performance of high efficiency particulate air (HEPA) filters under normal and abnormal conditions to establish criteria for calculating the efficiency of HEPA filters in a DOE nonreactor nuclear facility during and after a Design Basis Accident (DBA). This study is only applicable to the standard deep-pleated HEPA filter with aluminum separators as specified in ASME N509 [1]. Other HEPA filter designs such as the mini-pleat and separatorless filters are not included in this study. The literature review included the performance of new filters and parameters that may cause deterioration in the filter performance such as filter age, radiation, corrosive chemicals, seismic and rough handling, high temperature, moisture, particle clogging, high air flow and pressure pulses. The deterioration of the filter efficiency depends on the exposure parameters; in severe exposure conditions the filter will be structurally damaged and have a residual efficiency of $0 \%$. Despite the many studies on HEPA filter performance under adverse conditions, there are large gaps and limitations in the data that introduce significant error in the estimates of HEPA filter efficiencies under DBA conditions. Because of this limitation, conservative values of filter efficiency were chosen when there was insufficient data.
\end{abstract}

The estimation of the efficiency of the HEPA filters under DBA conditions involves three steps. In the first step, the filter pressure drop and environmental parameters such as temperature and moisture are determined during and after the DBA. The second step consists of comparing the filter pressure drop to a set of threshold values above which the filter is structurally damaged. There is a different threshold value for each combination of environmental parameters. The filter efficiency is determined in the third step. If the filter pressure drop is greater than the threshold value, the filter is structurally damaged and is assigned $0 \%$ efficiency. If the pressure drop is less, then the filter is not structurally damaged; and the efficiency is determined from literature values of the efficiency at the environmental conditions. The efficiency of the HEPA filters within DOE facilities should be determined on a case-by-case basis.

"This work was performed under the auspices of the U.S. Department of Energy by Lawrence Livermore National Laboratory under contract No. W-7405-Eng.48.

1. Harvard School of Public Health, 665 Huntington Ave., Boston, MA 02115

2 Consultant, R.R. 4, Box 4172, LaPlata, MD 20646

3. Consultant, P.O. Box 704, McLean, VA 22101

4. Consultant, P.O. Box 29720, Columbus, $\mathrm{OH} 43229$ 


\section{3rd DOE/NRC NUCLEAR AIR CLEANING AND TREATMENT CONFERENCE}

\section{Introduction}

The primary standard that governs the use of HEPA filtration systems in DOE facilities is DOE Order 6430.1A [2], which in turn refers to ASME N509 [1]. However, the standards do not provide guidance for determining their efficiency under abnormal or accident conditions. Under normal operating conditions, a HEPA filter will have a minimum efficiency of $99.90 \%$ [3]. This is the minimum filter efficiency at the most penetrating particle size of $0.15 \mu \mathrm{m}$ diameter for a HEPA filter that has a minimum efficiency of $99.97 \%$ for $0.3 \mu \mathrm{m}$ DOP particles. Previous publications had assigned a single value for the efficiency of HEPA filtration systems under all accident conditions. However the publications reviewed in this paper demonstrate that the efficiency of HEPA filters will vary greatly depending on the operating conditions, thereby requiring a case-by-case analysis.

Elder et al [4] prepared a guide for analyzing the accidental release of radioactive material from nonreactor nuclear facilities. This guide reviews the applicable DOE orders, provides a description of the design basis accidents, and evaluates the consequences of the accidents. In his section on reduction and removal factors, Elder discusses the efficiency of HEPA filters under DBAs. Elder recommended that the first stage HEPA be credited with an efficiency of $99.9 \%$ and each subsequent stage with $99.8 \%$. These values were selected from an unofficial HEPA filtration guideline that was established in 1971 during a meeting between officials from the Atomic Energy Agency and Albuquerque Operations Office [5]. These guidelines represented the opinions of the meeting attendees, and were not supported by technical data.

Walker [6] tabulated the available data on HEPA filter efficiency as of 1978 and recommended efficiencies of $99.9 \%$ for the first stage, $99.0 \%$ for the second and third stages, and $83.0 \%$ for the fourth stage. Although Walker cited experimental data, the recommended values were based on his opinion. Walker further stated in the summary that further study of HEPA filter efficiency is needed "to better establish relationships with relative humidity and temperature of sweep gas, service aging, material loading, etc."

The Nuclear Regulatory Commission in Regulatory Guide 1.52, recommended an efficiency of $99 \%$ for a filtration system consisting of a two stages of HEPA filters with an adsorber in between [7]. The guide is applicable to light-water-cooled nuclear power plants, where the filtration system is off-line during normal operations and only activated during accident conditions. Table 1 of the code shows that environmental conditions to which the HEPA filters would be exposed are approximately atmospheric pressure, $180^{\circ} \mathrm{F}, 100 \%$ relative humidity, and 105 rads/hr. There is no explanation for setting the HEPA

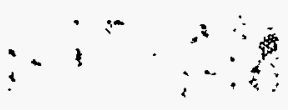




\section{3rd DOE/NRC NUCLEAR AIR CLEANING AND TREATMENT CONFERENCE}

filter efficiency at $99 \%$, when the new filter is tested at $99.97 \%$ for $0.3 \mu \mathrm{m}$ DOP particles and is also tested in-place for leaks at $99.95 \%$ as prescribed in ASME N510. [8]

The Nuclear Air Cleaning Handbook [9] recommended $99.8 \%$ efficiency for the first stage and $99.9 \%$ to $99.95 \%$ for each of the remaining stages. The Handbook presumably recommends a lower efficiency for the first HEPA filter and a higher efficiency for the second stage HEPA than the AEC 1971 draft because of the assumption that the first filter would take the brunt of any adverse effect from accidents. In discussing multistage HEPA filtration, the Handbook stated on page 38, "The purpose is to increase the reliability of the system by providing backup filters in the event of damage, deterioration, or failure of the first filters."

The major deficiency in the previous guidelines on HEPA filter efficiencies under DBA conditions is that the HEPA filter efficiency in DOE facilities can vary from $99.9 \%$ to $0 \%$ depending on which of the large number of different DBA conditions is applicable. In contrast, for light water reactors, there is a single DBA condition for which the HEPA filter efficiency is assigned 99\% [7] We have proposed a method for computing the efficiency of HEPA filters on a case-by-case basis using the available data reported in the literature. In this paper we describe the development of criteria for calculating the efficiency of HEPA filters during and after a DBA. These criteria are intended to be used in a future DOE Standard.

The goal of this paper is to provide guidance for computing the efficiency of HEPA filters during and after a DBA. The computed filter efficiency can be used in determining off-site doses from postulated releases of airborne radioactivity for both existing and future facilities. However, this study is not intended to define criteria for HEPA filter survival or provide guidelines on how to construct a HEPA filter that survives a DBA. Since there are a large number of different DBAs that are applicable to the many different DOE facilities, the HEPA filter efficiency will be computed on a case-by-case basis.

\section{Approaches for Computing HEPA Filfer Efficiency Under DBA}

\section{Single Efficiency Value for All DBA Conditions}

We considered various approaches for developing the criteria for computing HEPA filter efficiency under DBA conditions. The previous approach was to specify a single efficiency value for all conditions. We considered this to be unrealistic since the efficiencies of the available HEPA filters vary widely with different environmental parameters. Since there are no commercially available HEPA filters certified for 


\section{3rd DOE/NRC NUCLEAR AIR CLEANING AND TREATMENT CONFERENCE}

use in DOE facilities that can survive all of the postulated DBA conditions it is not possible to assign a single efficiency value to HEPA filters.

For the currently available HEPA filters, the only efficiency value that can be applied for all DBA conditions is $0 \%$. However, since the HEPA filters in most DOE facilities will survive the applicable DBA, assigning $0 \%$ efficiency to these filters is unrealistic. We concluded that until a high-strength HEPA filter is developed, it will not be possible to assign a single efficiency to HEPA filters that will apply for all DBA conditions.

\section{Efficiency Determined From Matrix of Three Severity Levels of Fire. Explosions, and Tornados}

We then reviewed various matrix approaches in which there are multiple filter stresses and multiple responses. A filter efficiency would be assigned for each of the filter stresses. In our initial review, there appeared to be only a few realistic DBA scenarios that include combinations of anticipated stresses that reflect graduated levels of severity. For example, a remote fire usually represents a filter loading episode without a very high temperature or a pressure pulse; an explosion usually represents a temperature and pressure pulse, and it may be the pressure resulting from rapidly increased airflow that does the damage and rapidly dies away to be followed by a fire without excessive pressure or airflow volume increase; a tornado results in a rapid pressure increase and/or decrease but no fire or particle/droplet loading. Each scenario can occur in varying degrees of severity that can probably be classified as "below concern", "moderate stress", and "extreme stress". This represents a $3 \times 3$ matrix and should not be excessively complex.

Although the concept is great, we were not able to assign an efficiency value for each of the proposed nine elements of the matrix because the three stresses of fire, tornado, and explosions were not single stress quantities, but could very widely in terms of more fundamental parameters such as temperature, pressure, and aerosol quantity and composition. For example estimating the efficiency of a HEPA filtration system after a fire cannot be derived from categories such as below concern, moderate stress or extreme stress. In order to assess the response to the fire, it will be necessary to estimate the temperature, quantity of smoke, type of smoke, water spray from a fire suppression system, the system flow rate, and the pressure drop across the HEPA filter. The pressure drop across the filter is, in turn, a function of the flow rate, water contact, and the quantity and type of smoke.

There is not a single DBA sequence that represents a fire. For example the remote fire scenario only involves filter clogging with little temperature increase or pressure pulse. In reality, there are a large 


\section{3rd DOE/NRC NUCLEAR AIR CLEANING AND TREATMENT CONFERENCE}

number of different fire scenarios: a well ventilated fire generating slow plugging aerosols, an under ventilated fire generating rapidly plugging aerosols, a fire with high temperature flames, use or non-use of fire suppression system based on water sprays and demisters, etc..

If the fire is remote and well ventilated, then the only consequence would be a small or moderate increase in pressure drop and no loss in efficiency. However a remote fire that is under ventilated (oxygen starved) would cause rapid filter plugging. If the air blower does not exceed the breaking point of new filters ( 37 inches ) then the effect would be a near or total shut down of the ventilation system. The efficiency of the clogged filter would be higher than the efficiency of the clean filter. If the smoke is diluted prior to reaching the HEPA filter, then the filter plugging will be reduced proportionately to the air dilution.

As another example dealing with fire, assume the fire is localized at the filter and reaches $400^{\circ} \mathrm{C}$. If no water suppression system is activated and the filter pressure drop is below 15 inches then the filter will be structurally undamaged but have a penetration about of about $3 \%$. However if the water spray system is activated, then the filter will rapidly plug due to water accumulation. If the air blower can pull

greater than 10 inches of water then the filter will be structurally damaged and have dramatically reduced efficiency (conservatively set at $0 \%$ ). However if the air blower cannot pull greater than 10 inches of water, then the plugged filter will shut down the ventilation system but still have the high efficiency.

Thus from these few examples we have shown that estimating the efficiency of the HEPA filters requires a more complex approach than categorizing a stress as below concern, moderate stress or extreme stress. There are also many other potential stresses than fire, tornado and explosion: for example, steam from a ruptured steam line, fire suppression system that sprays water, chemical effluents, and seismic stress.

In addition, the DBA stresses cannot be limited to a small number of different conditions because new DBA conditions will be established for new facilities and operations as DOE missions and directions change. New programs in weapons dismantlement, waste clean up, decontamination and decommission operations will bring their own set of stress factors.

\section{Efficiency Determined from Generalized Matrix of Operating Parameters and Parameters Causing} Structural Damage

We then evaluated a generalized matrix in which the efficiency is established at each level of stress for a series of fundamental parameters at increasing parameter level. Table 1 illustrates the 


\section{3rd DOE/NRC NUCLEAR AIR CLEANING AND TREATMENT CONFERENCE}

generalized matrix for tabulating the filter efficiency at each parameter level for a series of different parameters. These are the known parameters that have an effect on filter efficiency.

Table 1. Generalized matrix for establishing filter efficiency for operating parameters

Efficiency at Different Parameter Levels

Qperating Parameter

Low

Moderate

High

temperature

solid particle loading

water/liquid particle loading

air flow

Although Table 1 shows only three parameter levels (low, moderate, and high), the complete table could consist of many parameter levels, each level defined by specific values of the operating parameter. The primary advantage of the generalized parameter matrix over the three-stress matrix is that the parameters are fundamental parameters that can be uniquely defined. It is also possible to use semi-empirical equations for computing the efficiency due to the different parameters. Since the four parameters can be considered to act independently on the filter, the filter efficiency for combination of parameters can be computed from the efficiency for individual parameters.

The effect of the four operating parameters on the HEPA filter efficiency can be explained in terms of particle capture and particle loading theory and experiments. For HEPA filters, the maximum particle penetration is determined by the Brownian motion and interception capture mechanisms [10]. As the temperature is increased, the Brownian motion will increase while the interception mechanism remains constant. Thus higher temperatures will result in higher filter efficiencies. As the air flow increases, the capture by Brownian motion will decrease because of the decreased particle residence time in the filter. Since air flow has no effect on the interception mechanism, the net effect of increased air flow will be a lowering of filter efficiency. At exceedingly high air flows, the particle inertia becomes important and increases the filter efficiency. For solid particle loading, the particles form dendritic structures that capture additional particles, thereby increasing filter efficiency [11]. For liquid particles, the particles coalesces within the filter and decrease the void volume within the filter medium. The decreased void volume results in higher internal velocity and thus less residence time for Brownian motion [12]. Thus, as liquid deposits form in the filter, the aerosol penetration will increase. 


\section{3rd DOE/NRC NUCLEAR AIR CLEANING AND TREATMENT CONFERENCE}

Unfortunately the direct approach for computing the efficiency of HEPA filters under DBA conditions described above is not possible because the HEPA filters are frequently structurally damaged, which lowers the filter efficiency. For example as the temperature is increased, the efficiency increases. Figure 1 shows the decontamination factor ( $D F=1 /$ penetration) as a function of particle diameter for a deep-pleated HEPA filter with aluminum separators at increasing temperatures up to $200 \mathrm{C}$.

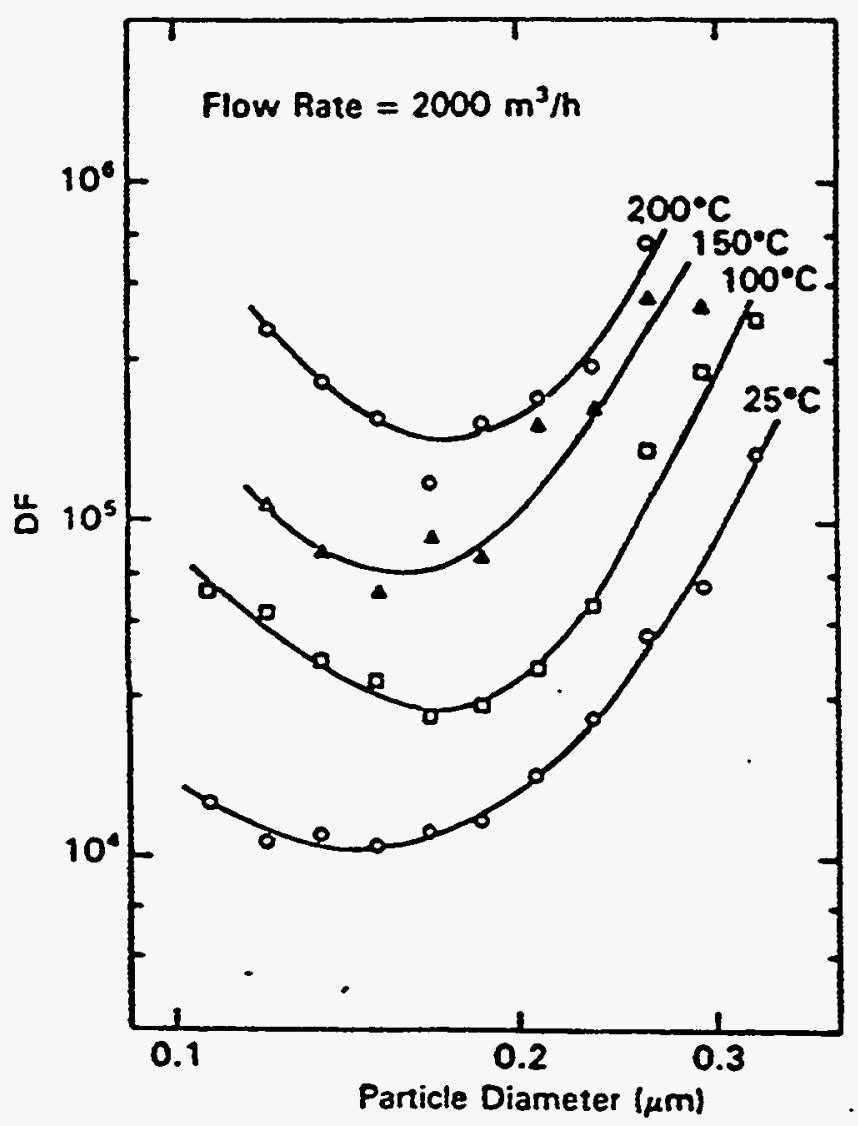

Figure 1 Decontamination factor (DF =1/penetration) for HEPA filter as a function particle diameter at increasing temperatures. Data from Osaki and Kanagawa [13].

With increasing temperature the organic components begin degrading and introduce leak paths through the filter. Figure 2 illustrates the effect of increasing temperature on the decontamination factor for HEPA filters with different frame materials.

The effect of increasing air flow provides another example of a stress having a direct effect on the filter efficiency due to the basic filtration mechanism and an indirect effect due to structural damage to the filter. Figure 3 shows the increasing DOP aerosol penetration as a function of particle size for a HEPA medium tested at increasing air velocities. 
23rd DOE/NRC NUCLEAR AIR CLEANING AND TREATMENT CONFERENCE

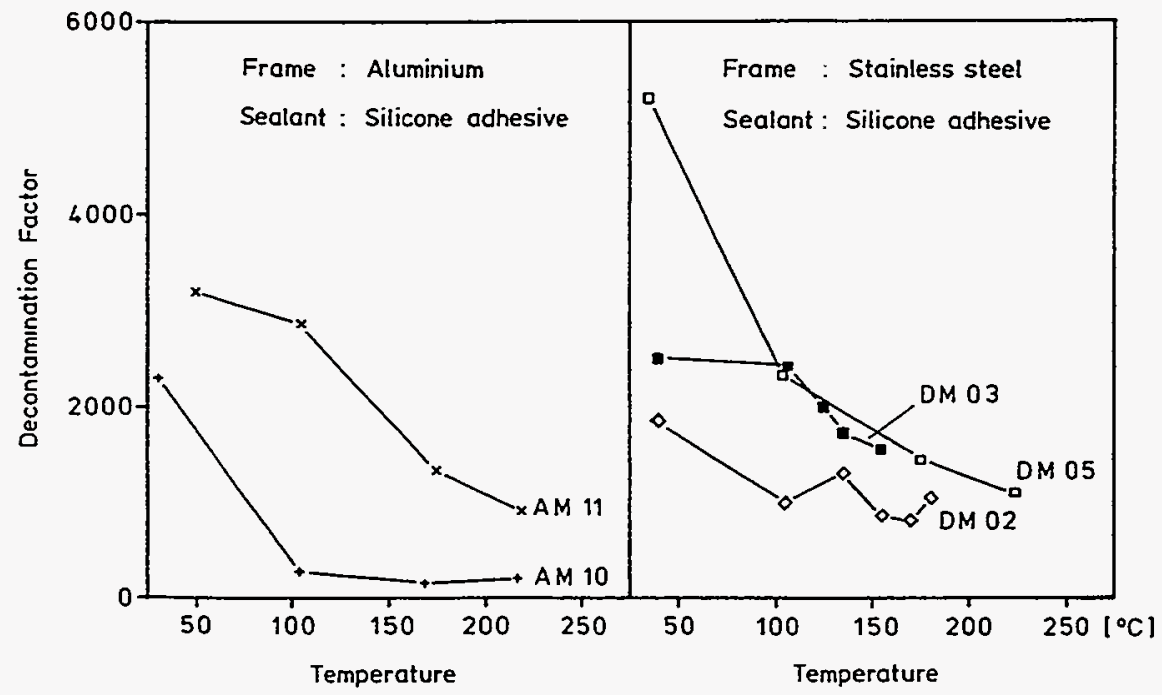

Figure 2 Decontamination factor for HEPA filters as a function of increasing temperatures. Data from Ensinger et al [14].

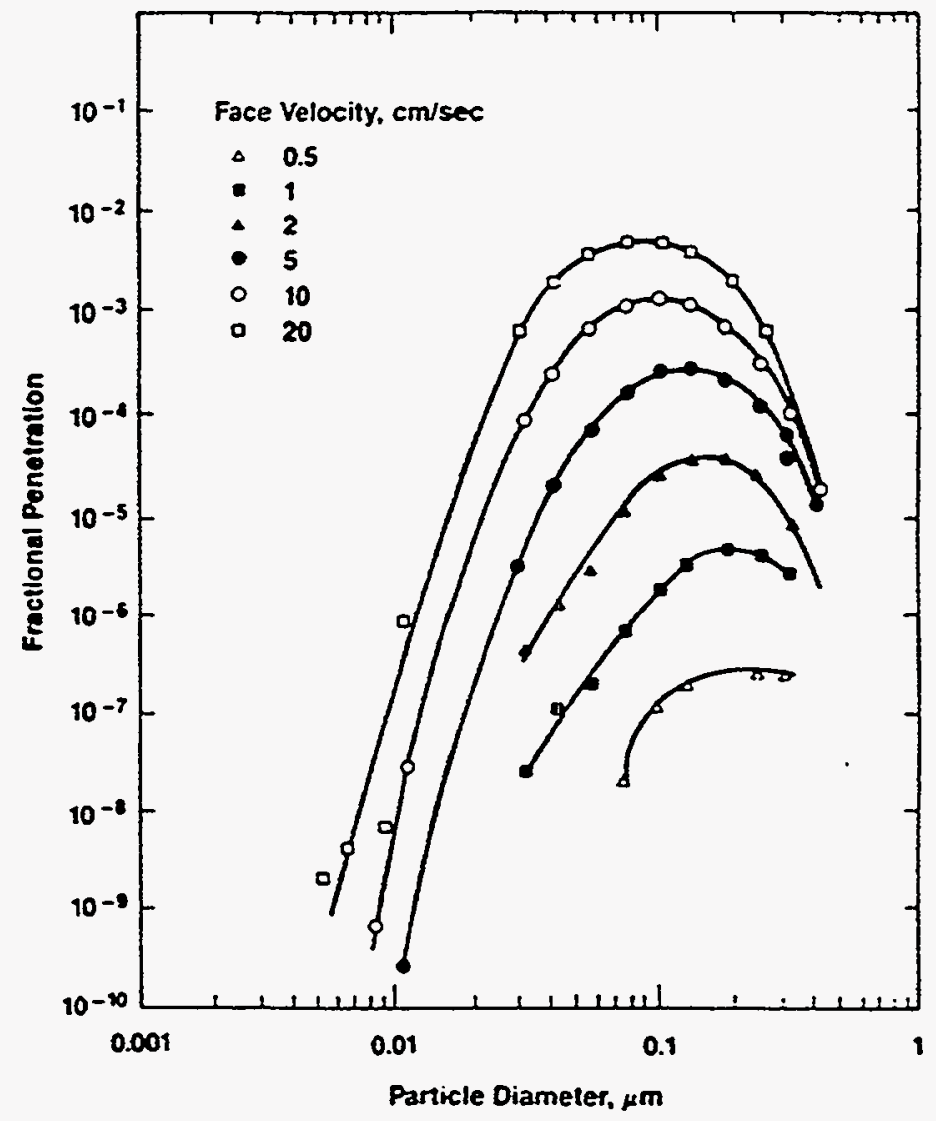

Figure 3. Penetration of DOP aerosols as a function of particle diameter at increasing air velocities for a HEPA medium, Lydall grade 213. Data from VanOsdell et al [15]. 


\section{3rd DOE/NRC NUCLEAR AIR CLEANING AND TREATMENT CONFERENCE}

As the air flow is increased the increasing pressure drop on the filter stresses the filter medium and eventually causes medium tears At high air flows, the entire medium pack is blown out of the housing as shown in Figure 4 . It is obvious that the fitter has $0 \%$ efficiency.

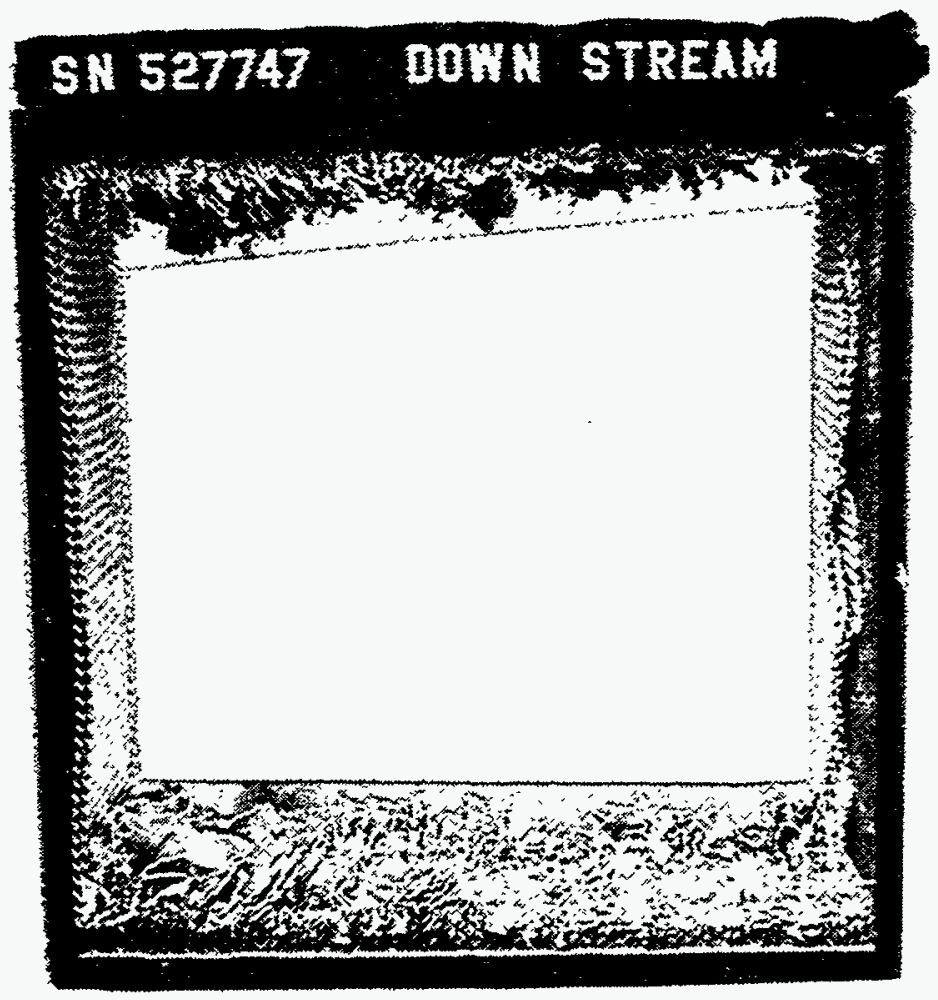

Figure 4 Photograph of 15-19 year old HEPA filter after subjected to a 13 inch differential pressure Photograph from Johnson et al [16].

There are additional stresses such as age, radiation exposure, water/liquid loading, seismic or rough handling, and chemical attack that also can weaken or cause structural damage to the HEPA fitter. Structural damage creates leak paths that lower the HEPA filter efficiency, whereas a weakened filter suffers structural damage at lower stress levels. Thus in order to determine the efficiency of HEPA filters under DBA conditions, it is necessary to include indirect effects due to HEPA filter damage in addition to the direct effects from the parameters listed in Table 1. However, incorporating structural damage and a weakened filter into a general method for determining filter efficiency is extremely difficult because a number of interacting parameters must be incorporated in the efficiency calculation. We will use data obtained from the literature to establish a method for computing the efficiency of HEPA filters under normal and DBA conditions. 


\title{
23rd DOE/NRC NUCLEAR AIR CLEANING AND TREATMENT CONFERENCE
}

\author{
111. Literature Review of HEPA Filter Efficiency Under Normal and DBA Conditions
}

The Minimum Baseline Efficiency for Each Stage of HEPA Filtration is $99.9 \%$

Single HEPA Stage According to ASME N509 each HEPA filter must have a minimum efficiency of $99.97 \%$ for $0.3 \mu \mathrm{m}$ DOP aerosols. However, recent studies have shown that the $0.3 \mu \mathrm{m}$ DOP aerosol is not the most penetrating size and therefore is not the most conservative efficiency. The particle penetration through HEPA filters is a strong function of particle size as shown in Figure 5 taken from Bergman et al [17]. The maximum particle penetration at $0.15 \mu \mathrm{m}$ diameter is about four times the penetration measured using $0.3 \mu \mathrm{m}$ DOP particles. The penetration decreases at smaller and larger particles due to competing particle capture mechanisms [18]. Particles less than $0.15 \mu \mathrm{m}$ have increasing Brownian motion with smaller sizes and therefore have lower penetration through the filter. Particles larger than $0.15 \mu \mathrm{m}$ have greater inertia with increasing size and are unable to follow the tortuous path through the filter. This results in a lower penetration with increasing size. The two capture mechanisms result in the experimental penetration curve seen in Figure 5.

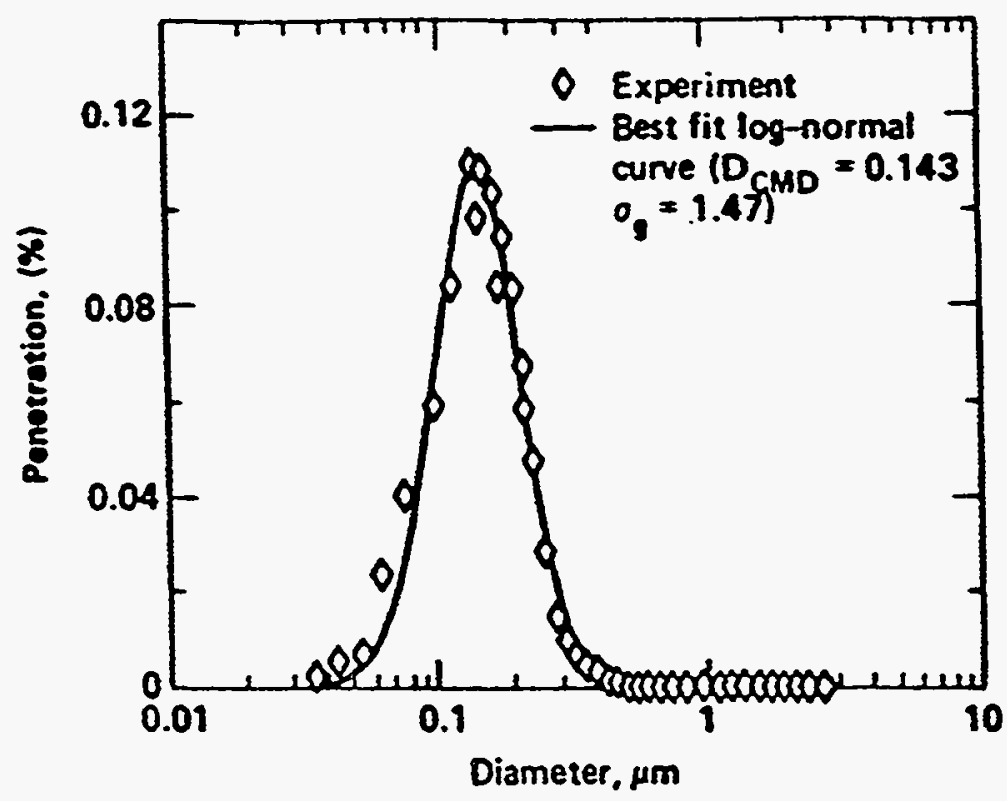

Figure 5 Penetration of DOS aerosols through a standard nuclear grade HEPA filter [17]. 


\section{3rd DOE/NRC NUCLEAR AIR CLEANING AND TREATMENT CONFERENCE}

Scripsick et al [3] conducted a comparison study between the standard DOP test using $0.3 \mu \mathrm{m}$ particles and the maximum penetration test in which penetration is measured as a function of particle size. They found that of 849 filters tested that had greater than $99.97 \%$ efficiency for $0.3 \mu \mathrm{m}$ particles, $18 \%$ of the filters had less than $99.97 \%$ but greater than $99.90 \%$ efficiency for $0.15 \mu \mathrm{m}$ particles. Thus the minimum efficiency for a new, undamaged HEPA filter is $99.90 \%$ at any particle size. The efficiency in most applications will be much greater, not only because the particle size is usually greater that $0.15 \mu \mathrm{m}$, but also because the particles generally have a greater density than the density of DOP $(0.98 \mathrm{~g} / \mathrm{cc})$. Anderson [19] and Tillery [20] have shown higher density aerosols have significantly higher efficiencies. However, if the aerosols consist of agglomerates of smaller particles, then the density will be significantly smaller than the bulk material density.

Multiple HEPA Filtration Stages. If the HEPA filter penetration is measured at the most penetrating particle size then the total maximum penetration for multiple stages of HEPA filters is the product of the penetration from each of the HEPA filters in series. There is no limit to the number of stages for which this computation will apply. However, each stage or combined stages must be leak-tested as specified by ASME N510 [8]. The penetration for each new, undamaged HEPA filter is $0.1 \%$ ( $99.9 \%$ efficiency). Untested HEPA filters with no visual damage are assigned a penetration of $10 \%$ ( $90 \%$ efficiency) based on the following field data using radiological measurements: Hetland and Russell [21] measured HEPA filter efficiencies of $94 \%$ and $83 \%$ for Pu aerosols in the second and third stages respectively. Frigerio and Stowe [22] measured an average efficiency of $99.4 \%$ for $\mathrm{Pu}$ and $92.9 \%$ for $\mathrm{U}$ in a third stage HEPA filter. Previously, untested HEPA filters were arbitrarily assigned a penetration of $0.2 \%$ ( $99.8 \%$ efficiency) $[9]$.

The determination of total penetration for multiple HEPA filters from the product of the individual filter penetrations appears to contradict the widely held belief that the filter penetration increases (i.e. efficiency decreases) with each filter stage. Previous studies that have shown a decrease in filter efficiency with multiple stages of filtration were generally based on average efficiencies for heterodisperse aerosols and/or have had significant background measurements. If filter efficiencies are based on average measurements over a particle size distribution, then the apparent filter efficiency will change with successive stages of filtration because the average particle size has changed. Ortiz [23] showed that even heterodisperse aerosols with narrow distributions will be altered when passing through HEPA titters and thereby cause a shift in the average particle size and the filter penetration.

Gonzales et al [24] conducted experiments with plutonium aerosols that showed the penetration of each HEPA filter in a system consisting of three filter stages is $99.99 \%$ for the first two stages and $99.84 \%$ for the third stage. They measured the HEPA penetration as a function of particle size for the first 


\section{3rd DOE/NRC NUCLEAR AIR CLEANING AND TREATMENT CONFERENCE}

two stages. The extremely low concentration of plutonium particles challenging the third filter made measurements extremely difficult and is most likely responsible for the lower fitter efficiency.

Osaki and Kanagawa [13] showed that the penetration of $0.15 \mu \mathrm{m}$ DOP particles for the second HEPA filter in a two-stage filter system yielded identical results as the first HEPA filter. They also verified that the two filters continued to have the same efficiencies at various air flows.

The study by Gonzales et al [24] and filtration theory were used in ERDA 76-21 [9] to conclude that the particle penetration through multiple HEPA filters equals the product of the penetrations of individual HEPA filters. ERDA 76-21 [9] points out that the multiplication of penetrations is valid for any particle particle size

\section{Literature Review of Parameters That Cause a Deterioration in HEPA Filter Efficiency}

A number of laboratory studies have shown that the efficiency of HEPA filters will degrade under certain environmental conditions. In extreme cases, the HEPA filter will be structurally damaged and result in $0 \%$ efficiency. The identified parameters that can cause a deterioration in the filter include age, moisture, chemical attack, high temperature, overpressure, shock and rough handling, particle loading, and radiation. The effect of these parameters on HEPA filters is dependant on the materials used and the design of the HEPA filters.

We summarized the literature data for the differential pressure required to cause structural damage on HEPA filters for various parameters in Table 2 in some cases only media data were available, and we had to compute the pressure drop for an equivalent HEPA filter. The percent reduction in media tensile strength or bursting strength from before and after exposure to a given parameter was used in the computation. This percent was then multiplied by the measured pressure drop required to blow out the medium in a new HEPA filter as measured by Gregory et al [25]. For example, Breschi et al [26] measured a 33\% reduction in medium burst pressure after exposure to $200 \mathrm{C}$ for one hour. The medium thus had $67 \%$ of its original strength. Multiplying $67 \%$ by 37 inches of water (the minimum pressure required to blow out the medium from a new HEPA by Gregory et al [25]) yields 25 inches of water. Although no data was available on the structural damage from acid exposure, the filter is degraded with increasing exposure and will even collapse by its own weight with prolonged exposure. 


\section{3rd DOE/NRC NUCLEAR AIR CLEANING AND TREATMENT CONFERENCE}

Table 2 Threshold Values of Differential Pressure Required to Structurally Damage the Standard HEPA Filter

\begin{tabular}{ll}
\multicolumn{2}{|c}{$\Delta \mathrm{P}$ threshold, } \\
\hline Avg. & Range \\
66 & $(37-81)$ \\
57 & $(38-72)$ \\
63 & $(47-90)$ \\
52 & $(29-70)$ \\
38 & $(13-78)$ \\
& \\
$33^{1}$ & $(18-40)^{1}$ \\
& \\
$X^{2}$ & \\
& \\
$44^{3}$ & $(25-54)^{3}$ \\
$33^{4}$ & $(19-41)^{4}$ \\
$26^{5}$ & $(15-32)^{5}$ \\
& \\
$13^{6}$ & $(8-16)^{6}$ \\
& $(8-20)$ \\
& \\
23 & $(10-36)$ \\
20 & $(16-25)$ \\
18 & $(7-36)$ \\
16 & $(3.6-25)$ \\
$40^{7}$ & $(22-49)^{7}$ \\
E0otnotes \\
\end{tabular}

Parameter

Baseline (high air flow)

Baseline (high air flow)

Baseline (high air flow)

Explosion shock

Age (15-19 year old filters

with Asbestos separators)

Radiation (5 $\left.\times 10^{7} \mathrm{rad}\right)$

$\mathrm{HNO}_{3}, \mathrm{HF}$ exposure (variable)

Temperature

$200^{\circ} \mathrm{C}\left(392^{\circ} \mathrm{F}\right), 1 \mathrm{hr}$.

$300^{\circ} \mathrm{C}\left(572^{\circ} \mathrm{F}\right), 10 \mathrm{~min}$.

$400^{\circ} \mathrm{C}\left(752^{\circ} \mathrm{F}\right), 1 \mathrm{hr}$.

$500^{\circ} \mathrm{C}\left(932^{\circ} \mathrm{F}\right), 10 \mathrm{~min}$.

$500^{\circ} \mathrm{C}\left(952^{\circ} \mathrm{F}\right), 10 \mathrm{~min}$.

Clean filter, water spray

Loaded filter, $100 \%$ humidity

Clean filter, water spray

Loaded filter, 99\% RH

Clean dry filter, prev. wet
Reference

Gregory et al [25]

Osaki et al [13]

Ruedinger et al [27]

Gregory et al [25]

Johnson et al [16].

Jones [28]

Woodard et al [29]

Breschi et al [26]

Hamblin et al [30]

Breschi et al [26] and Hamblin et al (30)

Pratt et al [31]

Pratt [32]

Ruedinger et al [33]

Ruedinger et al [33]

Ricketts et al [34]

Ricketts et al [34]

Ricketts et al [34]

1. Values computed from a measured $50 \%$ reduction in media tensile strength and base line values from Gregory et al [25].

2. No available data relating differential pressure threshold and acid challenge. Observations of HEPA filter after acid challenge show the HEPA media collapses and may fall out of its housing by its own weight.

3. Values computed from a measured $33 \%$ reduction in media rupture pressure and baseline values from Gregory et al [25].

4. Values computed from a measured $50 \%$ reduction in media rupture pressure and baseline values from Gregory et al [25].

5. Values computed from a measured $60 \%$ reduction in media rupture pressure and baseline values from Gregory et al [25]

6. Values computed from measurement of $80 \%$ reduction in tensile strength and baseline filter values from Gregory et al [25].

7. Values computed from measurement of $40 \%$ reduction in tensile strength and baseline filter values from Gregory et al [25].

Note: This table applies to HEPA filters having the deep-pleat design, organic sealant, and conventional glass fiber media. Other commercially available HEPA filters have lower threshold values for differential pressure. 
Table 3 Effect of Parameters on the Penetration of HEPA Filters

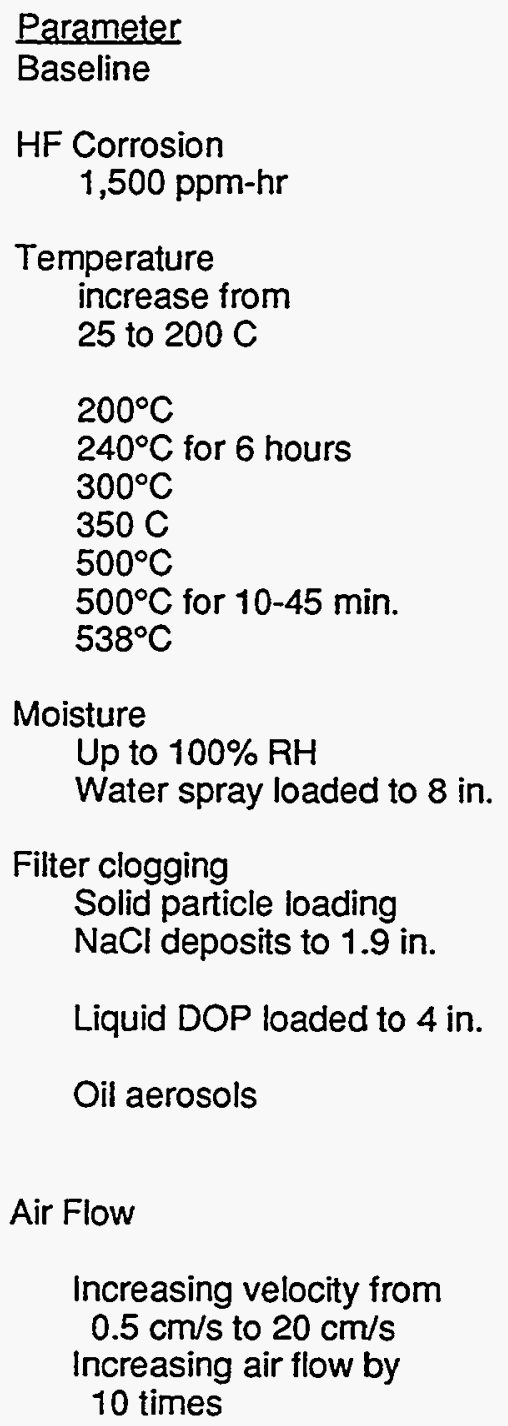

Filter clogging

Solid particle loading

$\mathrm{NaCl}$ deposits to 1.9 in.

Liquid DOP loaded to 4 in.

Oil aerosols

Air Flow

Increasing velocity from $0.5 \mathrm{~cm} / \mathrm{s}$ to $20 \mathrm{~cm} / \mathrm{s}$ Increasing air flow by 10 times

Air Pulse

1 psi pulse

Shock tests on filters preloaded with $.46 \mu \mathrm{m}$ latex

Seismic ( 0.2- $0.3 \mathrm{~g})$
Effect on Filter Penetration
$0.1 \%$

$0.1 \%$ increase

decreases penetration

from 0.01 to $0.001 \%$

$.03-0.01 \%$

$0.01 \%$

$0.12-0.01 \%$

$0.4-0.03 \%$

$0.9-0.2 \%$

$0.9-0.1 \%$

$1.2-0.5 \%$

Negligible effect

Increase by 10 times

Decreases penetration
Decreases penetration from
0.003 to $0.000001 \%$
Penetration increases
by factor of 10
Penetration increase is
$1.3 \mathrm{P} \Delta \mathrm{P} / \Delta \mathrm{P}_{\mathrm{i}}$ increase

Penetration increases from $0.00003 \%$ to $0.5 \%$

Penetration of $0.1 \mu \mathrm{m}$ particles increases by 100 times

Penetration of $0.46 \mu \mathrm{m}$ latex particles is $0.1 \%$

Penetration is $0.9 \%$

negligible effect
Reference

Scripsick et al [3]

Brassel et al [35]

Osaki et al [13]

Pratt et al [31]

Osaki et al [13]

Pratt et al [31]

Pratt et al [31]

Pratt et al [31]

Hackney [36]

First [37]

Osaki et al [13]

Osaki et al [13]

Bergman [11]

Osaki et al [13]

Osaki et al [13]

Payet et al [1.2]

VanOsdell et al [15]

Osaki et al [13]

Gregory et al [25]

Gregory et al [25]

Bergman et al [38]

We also reviewed the literature for the effect of different parameters on the efficiency of HEPA filters and have summarized the data in Table 3. The HEPA filters in these studies did not suffer structural damage except for the higher temperature and acid exposure tests. Although the HF exposure shows only a single entry, the penetration will increase with increasing exposure due to chemical attack 


\section{3rd DOE/NRC NUCLEAR AIR CLEANING AND TREATMENT CONFERENCE}

on the filter medium. Table 3 also shows a range of penetration values that were observed for different HEPA filters after the high temperature exposures.

\section{Developing a Method for Determining Filter Efficiency Under Normal and DBA Conditions}

Generalized Matrix Method is Not Feasible

Our initial approach was to incorporate the literature data on filter deterioration into the generalized matrix shown in Table 1. Unfortunately it is not possible to simply add additional parameters that weaken the filter or cause structural damage such as age and radiation exposure to the existing parameter list because the parameters are not independent. For independent parameters, it is not necessary to consider parameter interaction in computing filter efficiency. For example, when determining the filter efficiency under specific conditions of temperature, solid particle loading, water/liquid loading and air flow where there is no structural damage, one can add the contributions to filter efficiency by each of the three parameters. In practice the change in filter penetration ( 1 - efficiency) due to each parameter is added to the baseline penetration to yield the total penetration.

For dependent parameters it is necessary to consider the parameter interaction when computing filter efficiency. All of the parameters that affect filter strength or cause structural damage are dependent parameters because the filter damage or weakening from one parameter affects the amount of damage or weakening from a second parameter. For example the minimum pressure drop (or flow) required to rupture a new HEPA filter is 37 inches [25] whereas the minimum pressure drop for a 15-19 year-old filter is 13 inches [16] Both filters had $0 \%$ efficiency after the test. Intermediate pressure levels are required to burst HEPA filters less than 15 years old. A different series of pressure and age parameters define the limits for each efficiency level between $0 \%$ and $99.9 \%$. In his study of water effects, Ricketts [34] showed that a combination of factors leads to a greater loss in filter efficiency than the cumulative effect of the individual factors alone. This illustrates that filter efficiencies derived from studies on filter structural failures require combination of parameters and cannot be determined by individual parameters.

The primary difference in a matrix consisting of independent parameters for filter efficiency and dependant parameters for structural damage is the number of matrix elements required. For $\mathrm{N}$ independent parameters and 3 values of each parameter, the matrix consists of $3 \mathrm{~N}$ elements. For $\mathrm{N}$ dependent parameters and 3 values of each parameter, the matrix consists of $3^{\mathrm{N}}$ elements. The parameters that affect the HEPA filter efficiency directly are temperature, solid particle loading, water/liquid particle loading, and air flow. Of these, the solid particle loading is the only independent parameter since the other parameters can also cause structural damage and hence are dependent 


\section{3rd DOE/NRC NUCLEAR AIR CLEANING AND TREATMENT CONFERENCE}

parameters. The remaining dependent parameters are age, radiation exposure, seismic or rough handling, and chemical attack.. Thus for one independent and seven dependent parameters, the matrix consists of $3+3^{7}=1,380$ elements. For a matrix with 4 values for each parameter, the matrix would consist of $4+4^{7}=16,388$ elements. Even if the matrix were reduced to the minimum parameters of temperature, water loading, air flow, and age, the 3 value matrix would consist of 81 elements, and the 4 value matrix 256 elements. These are obviously very large matrixes.

We believe that it is not practical to pursue the development of a generalized matrix for computing the efficiency of HEPA filters under normal and DBA conditions because there are far too many elements, the experimental data is highly variable, and a separate matrix would be required for each design and construction of HEPA filter. Although a large number of studies are reported in the literature on the efficiency of HEPA filters under adverse conditions, all of the studies have been limited to one or two parameters, whereas most DBAs involve several parameters. There are few studies on evaluating filter efficiency for combination of parameters. As shown in our matrix examples, the large number of matrix elements is due to the combinations of different parameters.

In addition most of the literature data pertains to the standard deep-pleated HEPA filter using the standard glass fiber medium with aluminum separators. If other filter designs and/or other materials are used, then a different filter efficiency matrix is needed. There are a large number of different combinations of filter media, filter pleat configuration, sealant, separators, frame and gaskets. Each combination of components yield a HEPA filter with a unique set of responses to applied stress. Unfortunately, there exists very little data for other filter designs or other materials. The other filter designs occasionally used are the mini pleat, the cylindrical cartridge with radial pleats, the cylindrical cartridge made with standard deep pleats and media and the deep pleated separatorless filter. The use of glass cloth reinforced filter media dramatically increases the filter strength and the value of differential pressure required to structurally damage the HEPA filter.

The final problem for computing filter efficiency that applies to the matrix and all other methods is the variability of the data. For example, Johnson et al [16] exposed 15-19 year-old HEPA filters to simulated tornados and measured the differential pressure across the filters during the approximately 5 second air pulses. They tabulated the differential pressure at which the filter showed the first signs of damage and the pressure drop for total medium blow-out The data is retabulated here in Table 4

All of the six filters tested had the media blown out as shown in Figure 4 except for the filter in test 17. Although no efficiency measurements were made on the filters, we added a column in Table 4 for the estimated efficiency at the end of the test. Since the filters in tests 18-23 had no media left in the 
filter housing, the efficiency was $0 \%$. The filter in test 17 had the medium pack torn loose from the filter housing, but did not blow out at 77.8 inches pressure drop, which was the maximum applied stress during the test. A more powerful air pulse was needed to blow out the medium. We estimated the efficiency of the partially damaged filter at $98 \%$ based on efficiency measurements made on filters showing similar structural damage.

Table 4. Differential pressure for initial and total damage to aged HEPA filers from Johnson et al [16]

\begin{tabular}{|c|c|c|c|}
\hline \multirow[b]{2}{*}{ Test No. } & \multicolumn{2}{|c|}{ Differential Pressure (in. water) } & \multirow[t]{2}{*}{ Estimated Efficiency (\%) } \\
\hline & Initial & Total & \\
\hline 17 & 25.5 & 77.8 & 98 \\
\hline 18 & -- & 15.5 & 0 \\
\hline 19 & 3.9 & 18.8 & 0 \\
\hline 21 & 3.3 & 56.8 & 0 \\
\hline 22 & 3.3 & 13.6 & 0 \\
\hline 23 & 2.8 & 45.7 & 0 \\
\hline Average & 7.8 & 38.0 & \\
\hline
\end{tabular}

Table 4 illustrates the problem with trying to establish an efficiency value for a HEPA filter subjected to a given stress that causes structural damage. There is an initial stress value at which the filter shows the first indication of structural damage. For the six filters tested, the initial stress values varies from 2.8 to 25.5 inches of water and has an average of 7.8 inches of water. The initial damage consisted of a small segment of a fitter pleat torn open. Based on efficiency tests on other filters with similar damage, the efficiency at the initial damage was about $99 \%$. As increasing stress was applied to the filter, the efficiency would decrease until it reached $0 \%$ at total medium blow out. The differential pressure at total medium blow out ranges from 13.6 inches to greater than 77.8 inches with an average somewhat greater than 38 inches.

Experimental Measurements Under DBA Conditions is the Preferred Method. But There Are No Facilities

The preferred method for determining HEPA filter efficiency under DBA conditions is to conduct experiments on a scaled HEPA filtration system under controlled test conditions that simulate the DBA. The scaled down, $1,000 \mathrm{cfm}$ HEPA filtration system would be mounted in a test duct that would contain the desired air flow or air pulse, temperature, and water content. All of the components of the HEPA filtration system would be included such as prefilters, water deluge system, mist eliminator, and one or more stages of single HEPA filters. The test would consist of a series of exposures that simulate the 


\section{3rd DOE/NRC NUCLEAR AIR CLEANING AND TREATMENT CONFERENCE}

specific DBA. For example a simulation of a fire DBA might consist of a smoke challenge followed by a temperature increase and then a water deluge spray. The test filter could be preconditioned with particle loading, chemical exposure, seismic stress, etc. as needed to represent the exposure history for the DBA. Several repeat test would be conducted to establish the variability in the data.

The experimental approach has many advantages over a system based on determining the filter efficiency from a match with a catalog of test conditions and the corresponding efficiencies. The most important advantage is that the experimental approach will yield efficiencies for experimental conditions that match the DBA conditions as close as is experimentally possible. The problem with the catalog approach is that most of the available data is limited to HEPA filter efficiency for a single exposure parameter and a single or limited range of parameter values. This is in contrast to most DBAs that consist of a series of concurrent and sequential parameters. The catalog matching approach will undoubtedly require approximations and assumptions because the catalog will not contain the large number of matrix elements needed to fully describe the particular DBA. Moreover the exact filtration components and HEPA filters can be used in the simulation tests. In contrast, the catalog approach would undoubtedly contain data for different HEPA filters and system components. Another advantage is that conducting simulations of DBAs on HEPA filtration systems would be less costly than an attempt to develop catalogs of all the possible matrix elements in each of the many different matrixes that correspond to specific HEPA filtration systems.

Unfortunately, there is no test apparatus in the U.S. that can conduct DBA simulations or generate DBA matrix elements for HEPA filtration systems. A special test apparatus would have to be built for either application. The existing U.S. test equipment is limited to studying single parameters such as high air flow (New Mexico State U.), smoke loading (LLNL), and the quality assurance tests of heated air, overpressure, and rough handling (Rocky Flats). The quality assurance test equipment at Rocky Flats is designed for specific test conditions as part of the filter qualification prescribed in MIL-F-51068 [39] and cannot be varied to provide a range of DBA parameter values. Although the European countries have test equipment that can evaluate HEPA filters under a wide range of values for specific parameters, they do not have an apparatus that can expose HEPA filters to multiple parameters that simulate DBA conditions. Germany has a test rig that can expose HEPA filters to high humidities and water sprays at temperatures up to $151 \mathrm{C}$ and another system that can expose HEPA filters to high air flows. England and France have test rigs that can expose HEPA filters to various temperatures up to $400 \mathrm{C}$. Much of the data on HEPA performance under off-normal conditions was generated in these European facilities.

We Selected the Method Based on First Determining Structural Damage and Then Efficiency 


\section{3rd DOE/NRC NUCLEAR AIR CLEANING AND TREATMENT CONFERENCE}

Since it is not feasible to determine HEPA filter efficiency under DBA conditions using the generalized matrix method, and since no experimental facilities are available, we examined alternative approaches that could make use of the data base of HEPA filter performance under off-normal conditions. We focused our efforts on a method for separating out the many dependent parameters that affect filter strength or cause structural damage since these parameters are responsible for the large matrix for establishing HEPA filter efficiency. The filter efficiency would then be determined in two steps: first determine the filter efficiency for the parameters that cause structural damage and then add any contribution to the efficiency by the parameters in Table 1 for an undamaged filter.

The parameters that affect the filter strength and structure are temperature, waterliquid loading, air flow (pressure drop), age, radiation exposure, seismic or rough handling, and chemical attack. We have placed pressure drop in brackets following the air flow because the two parameters are directly proportional. Of these parameters only air flow (pressure drop) and seismic or rough handling can cause structural damage directly. The other parameters only weaken the filter. Thus higher air flows and resulting pressure drops will produce greater structural damage in the filter.

Particle loading is an important factor in determining the filter pressure drop, but is is not important for the threshold value of pressure drop at which the HEPA filter shows structural damage. A HEPA filter will suffer structural damage at the same pressure drop, whether or not particles are present. Particle deposits will increase the filter pressure drop and cause the threshold pressure drop to be reached at lower flow rates. However, the particle deposits will not lower the threshold pressure drop. Particle deposits were therefore not included in Table 5. None of the studies reviewed in this paper show that particle deposits have much of an effect on the structural strength of HEPA filters. Ricketts et al observed a $5 \%$ reduction in tensile strength of creased media [34]. Particle deposits also significantly increase the amount of water absorption on a filter, which subsequently decreases filter strength. However, this decreased strength is due to water, not particle deposits. Ricketts et al [34] found no significant difference in structural strength between HEPA filters with or without particle deposits during exposure to humid air and water sprays. Smith et al [40] observed a small increase in the pressure drop required to cause structural damage with HEPA filters loaded with salt particles. The salt deposits had formed a crust over the protective wire screen, thereby protecting the HEPA filter medium.

We wanted to construct a table showing filter efficiency at increasing levels of structural damage produced by increasing pressure drop for the parameters that weaken the filter. Unfortunately, the literature data contained little or no information on the efficiency of partially damaged HEPA filters. Most of the studies pertaining to HEPA filter damage only measured the pressure drop as shown in Table 2 


\section{3rd DOE/NRC NUCLEAR AIR CLEANING AND TREATMENT CONFERENCE}

and not the efficiency. The literature data was also limited to a single pressure drop measurement. In most cases, the pressure drop data corresponded to the threshold or first intermediate damage level. However, in a few cases, the pressure drop corresponded to the to total medium blow-out.

The level of structural damage is important since the efficiency can vary from $99 \%$ for a partially damaged filter to $0 \%$ for a totally damaged filter. Generally, the reported pressure drop data on structural damage due to high air flow corresponded to a partially damaged HEPA filter. Johnson et al [16] reported the pressure drop corresponding to both the threshold and the medium blow-out as shown in Table 4 . The threshold and medium blow-out data were derived from a motion picture camera synchronized with the pressure pulse of a simulated tornado. In all of the other studies, only the final condition of the filter was available. Unfortunately, except for the high temperature studies, few efficiency measurements were made on the partially damaged filters, and few quantitative descriptions of the filter damage were made.

Because of the limitations in the literature data, we selected the method of first determining the structural damage on the HEPA filter and then the efficiency. If the HEPA filter suffered significant structural damage, the filter would be assigned $0 \%$ efficiency. If the filter was not significantly damaged, then the efficiency would be determined from literature values of the efficiency.

Justification for Assigning 0\% Efficiency To Partially Damaged Filters: The selection of the filter efficiency that corresponds to the reported pressure drop for partially damaged HEPA filters in Table 2 was a major step in the development of a method for computing filter efficiency under DBA conditions. We decided to set the efficiency for all damaged HEPA filters, whether partially or fully, to $0 \%$ for several reasons. First, there is very little data on the efficiency of partially damaged HEPA filters. The few reported efficiency measurements show partially damaged HEPA filters have high efficiencies. For example, a HEPA filter with a 5 inch slit along one pleat of the HEPA medium has an efficiency of $99 \%$, while a filter that has the media pack loose within the frame and slightly pushed out of the frame has an efficiency of $94 \%$ [41]. The primary reason for selecting $0 \%$ efficiency for partially damaged HEPA filters is that there is a large variability in the differential pressure required to blow out the filter medium as seen in Table 4 for high air flow (from 13.6 to greater than 77.8 inches). This variability makes it prudent to select a conservative value of differential pressure at which the filter medium blows out of the filter.

Another important reason for selecting conservative values is that most DBA s consist of multiple challenges, whereas the available data generally applies to one parameter, not counting the differential pressure, that is responsible for the damage. We have assumed that using conservative values for the pressure drop that is required to blow out the filter medium due to a single parameter will compensate for the additional damage caused by multiple parameters. 


\section{3rd DOE/NRC NUCLEAR AIR CLEANING AND TREATMENT CONFERENCE}

Table 5 Threshold Values* of Differential Pressure Required to Structurally Damage the Standard HEPA Filter

\section{Parameter}

Baseline (new filter, normal, conditions)

Age (15 years or older)

Radiation $\left(6 \times 10^{7} \mathrm{Rad}\right)$

Chemical $\left(\mathrm{HNO}_{3}, \mathrm{HF}\right)$

Temperature

less than $200^{\circ} \mathrm{C},\left(392^{\circ} \mathrm{F}\right)$

$200-300^{\circ} \mathrm{C},\left(192-572^{\circ} \mathrm{F}\right)$

10 minutes

1 hour

10 hours

$300-400^{\circ} \mathrm{C},\left(572-752^{\circ} \mathrm{F}\right)$

$400-500^{\circ} \mathrm{C},\left(752-932^{\circ} \mathrm{F}\right)$

\section{$\Delta P$ Threshold" inches w.g.}

37

13

18

$0-37$

37

33

30

22

15

10

10

22

29

Air pulse from explosion

wet filter, (greater than $95 \%$ relative humidity) dry filter, previously wet

- These values represent the most conservative values (except for moisture) taken from an analysis of experimental studies reviewed in this report and summarized in Table 2

We also did not select the lowest value for structural damage at $500 \mathrm{C}$ because 8 inches only produced tears in the media; but did not cause total filter blow-out [32]. In a second test, Pratt [32] observed a total filter collapse at 20 inches after exposure to $500 \mathrm{C}$. We selected 10 inches of water as a compromise between the conservative value based on partial filter damage in one of the two tests conducted by Pratt [32] and the wide range in values observed in Table 2 for HEPA structural damage.

It is important to note that the parameters in Table 5 represent exposures to a HEPA filter during its life, not just during the DBA. All of the parameters must be taken into account throughout the life of the filter in addition to the parameters applicable to the specific DBA. The damage caused by the different parameters is not reversible except for water, and that is only partially reversible. A previously wet HEPA filter will have a $40 \%$ reduction in its tensile strength [34].

A major concern with the use of Table 5 for determining filter survivability is the question of whether multiple stresses act independently and are additive. Unfortunately there are not sufficient data available to answer this question definitively. Nearly all previous studies on HEPA stress were done with single stresses. However, it is possible to make general observations. Age, radiation and temperature 


\section{3rd DOE/NRC NUCLEAR AIR CLEANING AND TREATMENT CONFERENCE}

all reduce the tensile strength of the filter medium. If a combination of these stresses were applied to the medium, the tensile strength of the medium would decrease up to the limiting value where the binder that holds the glass fibers together is no longer effective. Thus the three parameters can be viewed as acting independently and can be added up to the limiting strength of no binder. The three stresses also cause a loss of water repellency, which makes the filter absorb water and loose strength. Since all of the stresses in Table 5 degrade the medium strength by attacking the binder, there is a lower strength limit for these parameters. An approximate value is 10 inches of water.

In contrast, the attack by chemicals such as $\mathrm{HNO}_{3}$ and $\mathrm{HF}$ attack the glass fibers directly and can continuously degrade the media strength to the point where it collapses under its own weight. Chemical attack must be treated as a separate case from the other stresses.

The effect of temperature on the structural damage to the HEPA filter depends on both the temperature value and the exposure time. Figure 6 shows the percent of the initial tensile strength and burst strength for HEPA filter media samples after ten minute exposures at the indicated temperatures [30]. The burst strength and tensile strength follow a common curve because the filter media are held together with similar acrylic binders [30]. Breschi et al [26] found that at $200 \mathrm{C}$, the filter medium becomes

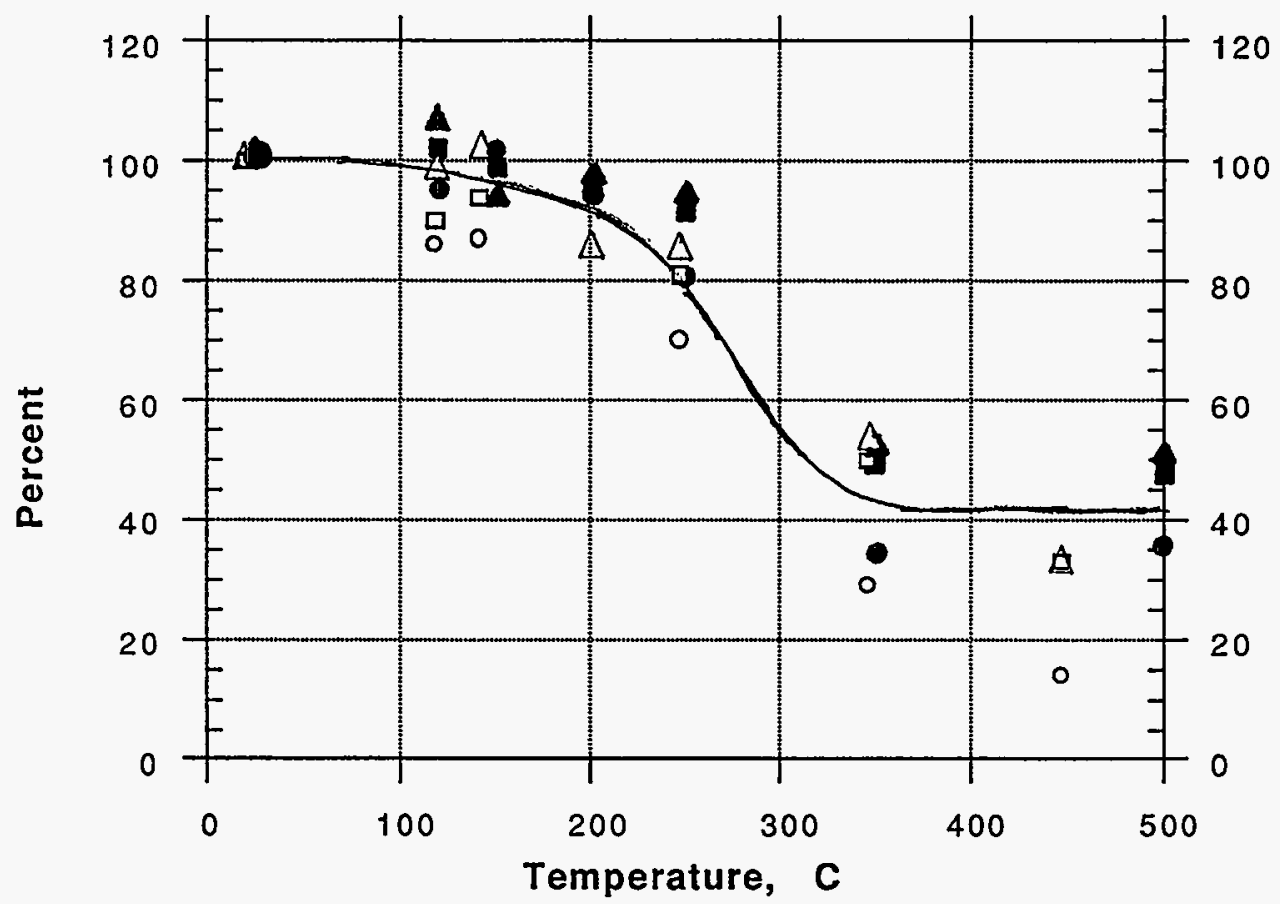

Figure 6. Percent of initial tensile strength and burst strength for three different HEPA media as a function of temperature. Open points represent tensile strength and solid points represent burst strength. Data replotted from Hamblin and Goodchild [30]. 


\section{3rd DOE/NRC NUCLEAR AIR CLEANING AND TREATMENT CONFERENCE}

brown, and electron micrographs show the microfibers are covered with small lumps and deposits due to melting of the binder. Raising the temperature to $300-400 \mathrm{C}$ cleans the microfibers from all deposits [26]. Gilbert et al [41] also found that the acrylic weight loss occurs between 300-400 C.

Figure 7 shows the percent of the initial tensile strength and burst strength as a function of time for HEPA filter media samples exposed to $120 \mathrm{C}$ and $250 \mathrm{C}$ [30]. At $120 \mathrm{C}$, the media show a negligible decrease in strength over time. However, at $250 \mathrm{C}$, the media show a gradual, but significant, loss in strength. Hamblin and Goodchild [30] also found that all the binder is lost in less than 10 minutes at $400 \mathrm{C}$.

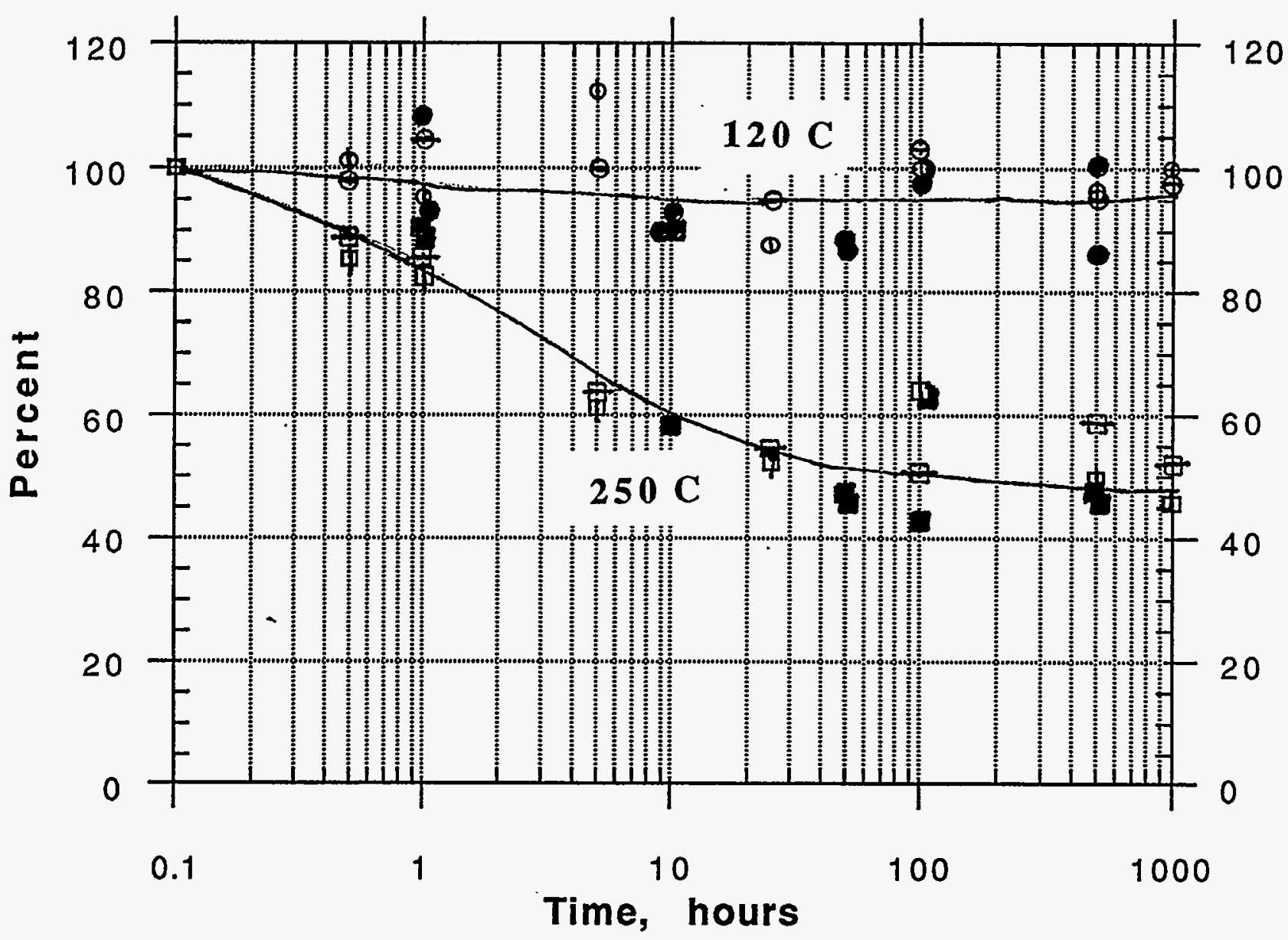

Figure 7. Percent of initial tensile strength and burst strength for three different HEPA media as a function of time after exposure to $120 \mathrm{C}$ or $250 \mathrm{C}$. Open points represent tensile strength and solid points represent burst strength. Data replotted from Hamblin and Goodchild [30]. 


\section{3rd DOE/NRC NUCLEAR AIR CLEANING AND TREATMENT CONFERENCE}

We have used these studies to generate the temperature and time data in Table 5. Since there is no significant deterioration at temperatures below $200 \mathrm{C}$, the threshold value of differential pressure will be the same as the baseline filter. For temperatures between $200-300 \mathrm{C}$, we used the curve for $250 \mathrm{C}$ in Figure 7. The estimated percent of initial strength at 10 minutes, 1 hour, and 10 hours are approximately $90 \%, 80 \%$, and $60 \%$ respectively. These percent values were multiplied by the baseline differential pressure of 37 inches and yield 33,30 , and 22 inches respectively. For temperatures above $300 \mathrm{C}$ the deterioration in medium strength is essentially complete within ten minutes as seen in Figure 6 . Thus, the differential pressure for temperature exposures between $300-400 \mathrm{C}$ is computed from $40 \%$ residual strength and the baseline 37 inches to yield 15 inches. Since the deterioration occurs in less then 10 minutes, there is no time associated with this exposure.

The temperature can also cause the sealant that holds the media pack in the frame to char and sometimes burn and break loose from the filter frame. High temperature can also cause the fitter medium to develop tears, especially when using steel frames due to the difference in thermal expansion between the steel frame and the medium pack. The additional failure modes lower the differential pressure for structural failure. These type of failure modes are not significantly affected by the other parameters in Table 5

Thus for determining the threshold differential pressure for structural damage, we can use the following prescription: For single stresses, the threshold differential pressure is read from the applicable entries in Table 5. For multiple stresses, whether sequential or simultaneous, the lowest differential pressure threshold is selected. If the environmental parameters are not well defined for a given DBA then the threshold value of 10 inches is used.

In order to use Table 5 for estimating structural damage, the pressure drop for a particular filter must first be determined for the environmental exposure in the DBA. The driving force for the filter differential pressure may be a fan, a tornado, an explosion, a pressurized gas release, or other sources of negative or positive pressures. For a plugged filter, the differential pressure can be approximated by the source pressure since nearly the full load will be applied across the filter. Cases were the filter may approach full plugging are during high smoke concentrations from oxygen starved fires [42] and water exposure on clogged filters [33,34].

For a clean or partially plugged filter, the differential pressure will be less than the source pressure and must be estimated. The most common source of partial filter plugging is particle deposits from atmospheric dust or plant operations. The data compiled by First and Price [43] and Novick [44] can be used to approximate the particle loading on HEPA filters. All new HEPA filters must have a pressure 
drop less than one inch of water at $1,000 \mathrm{cfm}$. At higher air flows the pressure drop increases as shown in Figure 8. This figure can be used directly to determine the pressure drop across a clean filter if the air flow is known. Figure 8 can also be used for computing the pressure drop of partially plugged fitters at a second flow if the filter pressure drop is known at one flow.

$$
\Delta P_{2}=\Delta P_{1} \times \Delta P_{C_{2}} / \Delta P_{C_{1}}
$$

where $\quad \Delta P_{2}$ is the pressure drop of the partially plugged fitter at the second flow

$\Delta P_{1}$ is the pressure drop of the partially plugged fitter at the first flow

$\triangle \mathrm{PC}_{1}$ is the pressure drop of the clean filter at the first flow from Figure 8

$\triangle P_{C 2}$ is the pressure drop of the clean filter at the second flow from Figure 8

For an illustration, assumie that a HEPA filter is loaded with particles and has a pressure drop of 4 inches at the standard flow of $1,000 \mathrm{cfm}$. If the flow suddenly increases to $10,000 \mathrm{cfm}$, the resulting pressure drop would be 48 inches $(4 \times 12 / 1)$. We obtained the 12 inches and 1 inch from Figure 8.

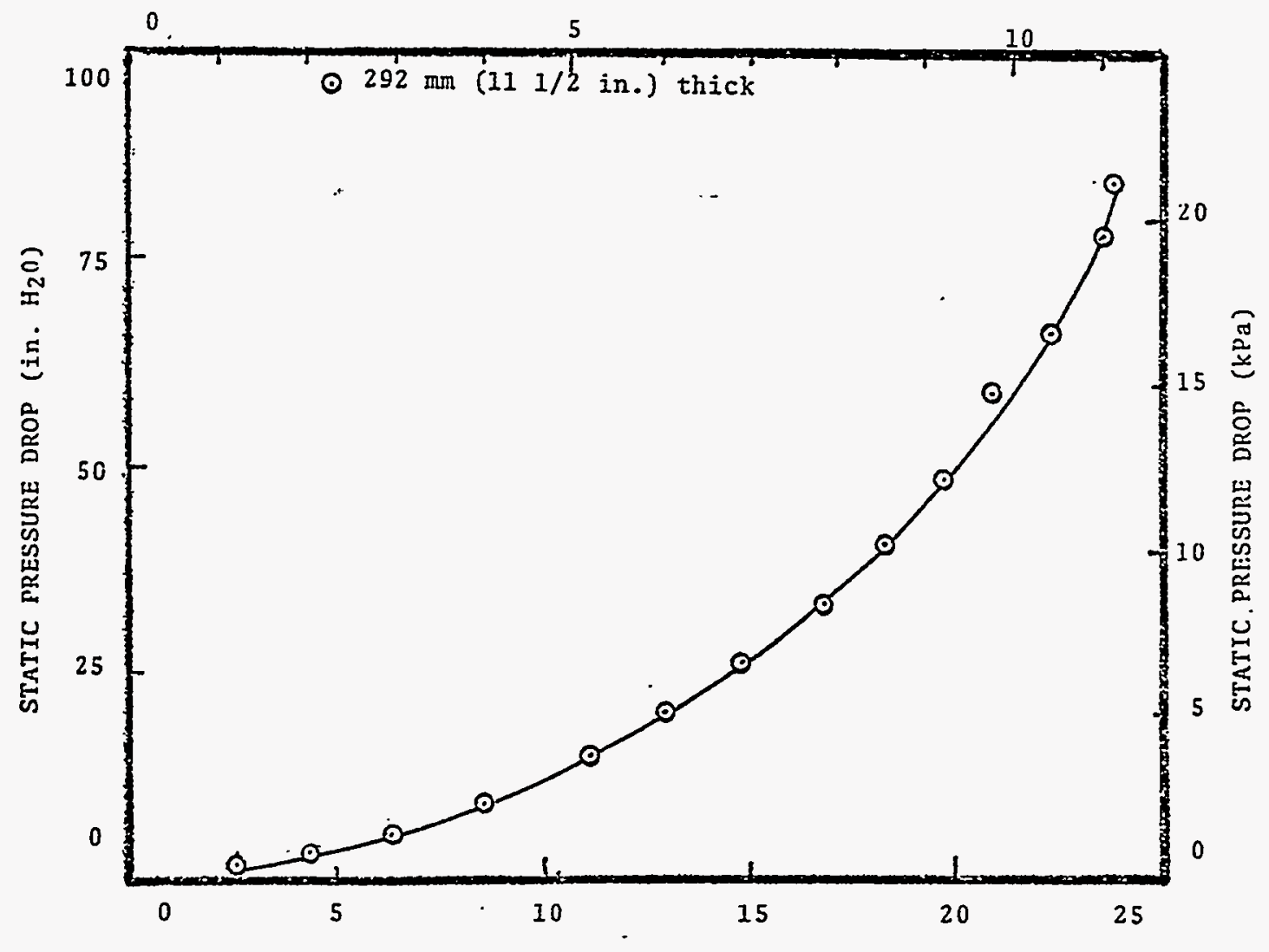

Flow, $\mathrm{ctm} \times 1,000$

Figure 8. Pressure drop as a function of flow rate for a clean HEPA filter from Gregory et al [45]. 


\section{3rd DOE/NRC NUCLEAR AIR CLEANING AND TREATMENT CONFERENCE}

Once the appropriate pressure drop is determined for the HEPA filter under DBA conditions, the value can be compared to the applicable threshold values listed in Table 5 . If the estimated differential pressure is less than the threshold value, then the HEPA filter will not be structurally damaged, and the filter efficiency can be determined in the next section. However, if the differential pressure exceeds the threshold values, then the filter will be structurally damaged and will be assigned a $0 \%$ efficiency under DBA conditions.

We can also use Figure 8 and Equation 1 to compute the air flow through a partially plugged filter if we know the initial pressure drop and flow rate and the final pressure drop. This computation is made easier by rearranging Equation 1 as follows:

$$
\Delta P_{C 2}=\Delta P_{C 1} \times \Delta P_{2} / \Delta P_{1}
$$

For example, if the partially plugged HEPA filter has a pressure drop of 4 inches at 1,000 cfm and is then subjected to a pressure drop of 48 inches, the pressure drop for the clean filter will be 12 inches $(1 \times 48 / 4)$. The corresponding air flow is then read from Figure 8 to be $10,000 \mathrm{cfm}$.

Once the appropriate pressure drop is determined for the HEPA filter under DBA conditions, the value can be compared to the applicable threshold values listed in Table 5 . If the estimated differential pressure is less than the threshold value, then the HEPA filter will not be structurally damaged, and the filter efficiency can be determined in the next section. However, if the differential pressure exceeds the threshold values, then the filter may be structurally damaged and will be assigned a $0 \%$ efficiency under DBA conditions.

\section{Determination of HEPA Filter Efficiency Under DBA. If the HEPA filter is not structurally} damaged, then the fitter efficiency can be estimated from the filter efficiency tables and from the conditions during the DBA. Table 3 summarizes the effect of various parameters on the penetration of HEPA filters obtained from our literature review. However, before the data can be used for determining filter efficiency under DBA conditions it must be consolidated and simplified.

One consolidation of Table 3 is to treat the air flow and air pulse as one parameter since they are not independent. This is possible because the particle capture mechanism is the same for both processes. In our combined air flow and pulse, the air pulse is viewed as a higher flow for a short time. We were also able to derive an empirical equation for the maximum filter penetration as a function of air flow based on the data from VanOsdell et al [15]. The maximum penetration point for velocities of $0.5,1$, 
$2,5,10$, and $20 \mathrm{~cm} / \mathrm{s}$ were taken from Figure 3 and regraphed in Figure 9 . The straight line through the data points fit the following equation:

$$
P=3.65 \times 10^{-4} \mathrm{~V}^{2.58} \% \text {, }
$$

where $P$ is the penetration in percent and $V$ is the velocity in $\mathrm{cm} / \mathrm{s}$.

Since air flow is a more practical unit of measure than air velocity through the medium, we can substitute air flow/filter area for velocity in Equation 3. If we assume the average deep-pleated HEPA filter has 200 square feet of media then Equation 3 becomes

$$
P=3.65 \times 10^{-4}(\text { flow } / 400)^{2.58} \% \text {, }
$$

where flow is in cfm.

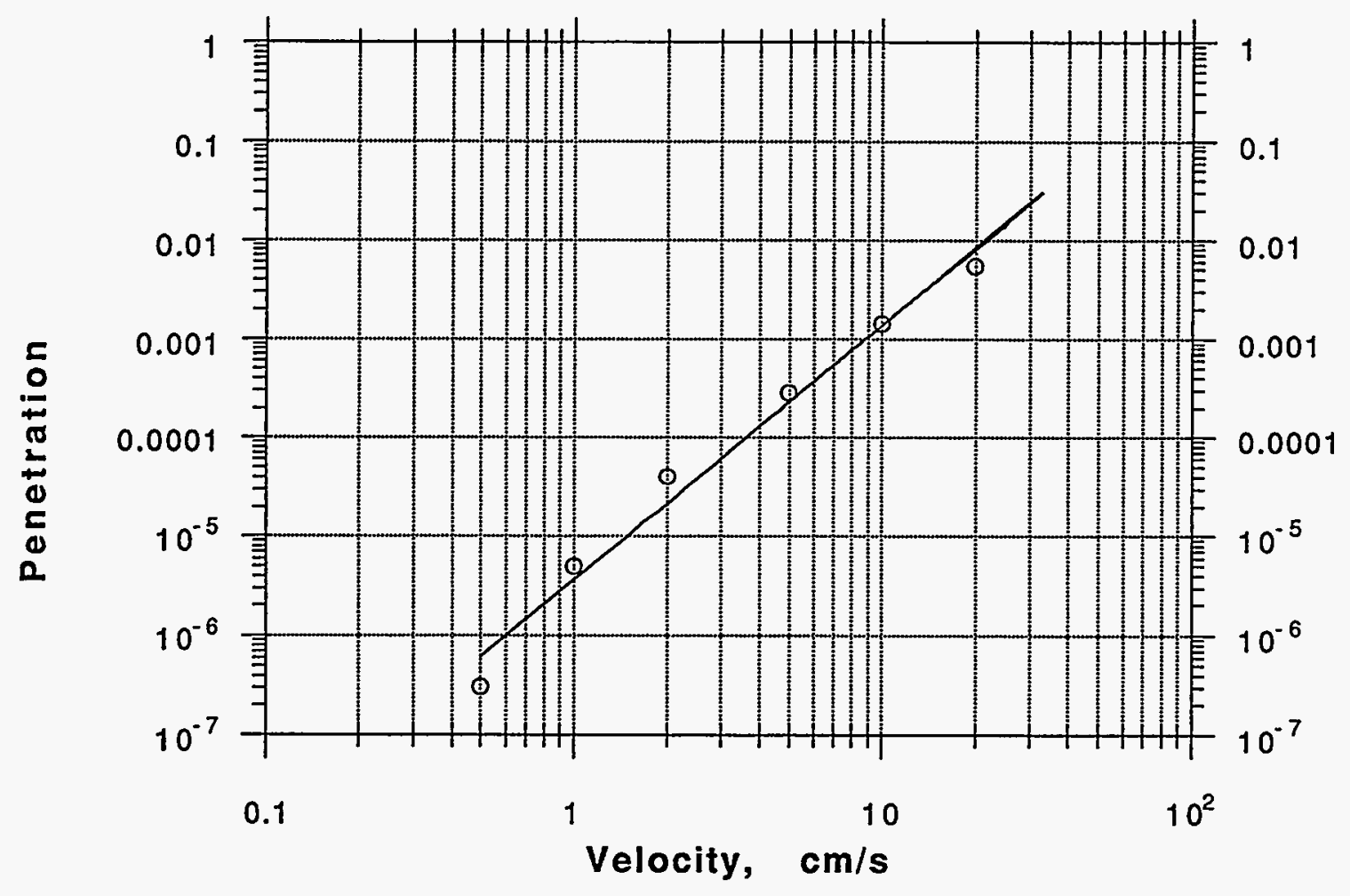

Figure 9. Maximum filter penetration as a function of air velocity replotted from Figure 3

Equation 4 also applies to an air pulse. However, in order to obtain filter penetration from the equation, it is necessary to determine the air flow through the filter that corresponds to the applied differential pressure. For example, a $1 \mathrm{psi}$ (27.7 inches) air pulse applied to a clean fitter corresponds to 


\section{3rd DOE/NRC NUCLEAR AIR CLEANING AND TREATMENT CONFERENCE}

an air flow of $15,000 \mathrm{cfm}$ as determined from Figure 8. Computing the penetration at $15,000 \mathrm{cfm}$ with Equation 4 yields a penetration of $4.2 \%$. Gregory measured $0.1 \%$ penetration for $0.46 \mu \mathrm{m}$ latex particles in a 1 psi pulse simulating a tornado [25]. He also measured $0.9 \%$ penetration for previously deposited $0.46 \mu \mathrm{m}$ latex particles in a simulated explosion shock ( $1 \mathrm{psi}$ estimated). Since the penetration for $0.1 \mu \mathrm{m}$ particles is more than ten times the penetration for $0.46 \mu \mathrm{m}$ particles (see Figure 5), Equation 4 is in general agreement with experiment.

We should point out that the increasing penetration with increasing air flow is not in disagreement with previous studies that show an initial increase and then a decrease in penetration with increasing air velocity [18]. In the previous studies, the particle size remained constant as the air flow increased. The penetration initially increased because of decreasing time for diffusional capture. With increasing air flow, the particle inertia increased and resulted in decreasing penetration. The observed penetration increased and then decreased as the dominant capture mechanism switched from diffusion to inertia. However, for the maximum penetration, the particle size decreases with increasing air velocity and therefore extends the range where diffusion dominates and results in increasing penetration with increasing air flow. The increasing contribution due to inertia is also seen in Figure 9 by a small deviation from the straight line to lower penetration levels.

We then prepared Table 6 for computing filter efficiencies under DBA conditions by consolidating the data from Table 3 and making simplifying assumptions. The primary assumption was to use the most conservative penetration value for each parameter. We also combined the penetration increase due to water accumulation and oil accumulation into a single liquid accumulation term. All of the entries in Table 6 represent penetration values to be added to the baseline $0.1 \%$ penetration. Equation 4 was used in place of the data for air flow and air pulses. Although the entry for HF corrosion shows only a single data, the penetration increases continuously with increasing HF exposure. Unfortunately there were no additional data available. For the temperature-parameter, we reduced the number of temperatures and used the highest penetration value. No credit was given for the initial decrease in penetration from 25 to $200 \mathrm{C}$, as shown in Figure 1, because the change in penetration was small compared to $0.1 \%$. We also used the maximum allowed penetration of $3 \%$ for exposure to $371 \mathrm{C}(700 \mathrm{~F})$ for 5 minutes as specified in MIL-F-51068 [39].

The final entries to complete Table 6 were solid and liquid loading. For solid loading we indicated $-0.05 \%$ since it improves filter efficiency. For liquid aerosols, the penetration increases by 1.3 times the product of the initial penetration and the ratio of the final pressure drop to the initial pressure drop. The increased penetration is due to liquid build-up that blinds portions of the medium and hence increases the 
air flow through the remaining unblocked portions of the medium. The higher air flow causes the increased particle penetration.

Table 6 Effect of Environmental and Filter Parameters on the Aerosol Penetration through HEPA filters.

Parameter

Baseline

HF corrosion

1,500 ppm-hr.

Temperature

less than $200^{\circ} \mathrm{C}\left(392^{\circ} \mathrm{F}\right)$

$200-300^{\circ} \mathrm{C}\left(392-572^{\circ} \mathrm{F}\right)$

$300-350^{\circ} \mathrm{C}\left(572-662^{\circ} \mathrm{F}\right)$

$350-500^{\circ} \mathrm{C}\left(662-932^{\circ} \mathrm{F}\right)$

Particle Deposits

Solids

Liquids

Air Flow (Air Pulse)

\section{Filter Penetration*}

$0.1 \%$

$0.1 \%$

$0.0 \%$

$0.1 \%$

$0.4 \%$

$3.0 \%$

- These values represent the most conservative values taken from an analysis of experimental studies reviewed in this report and summarized in Table 3.

Table 6 can be used to estimate the aerosol penetration of a HEPA filter under the conditions expected in a given DBA. Once the parameter values that apply for a given DBA are established, the corresponding penetrations can be used for determining the HEPA filter penetration. As we have previously discussed, since the penetrations due to the different parameters are independent of each other, then the filter penetration for a combination of parameters will equal the sum of the penetrations for the individual parameters.

Thus for determining the aerosol penetration from the data in Table 6, we use the following prescription: For single stresses, the penetration is read from the applicable entries or computed from the flow. For multiple stresses, the penetration from each of the stresses are added to yield a total filter penetration. 


\section{3rd DOE/NRC NUCLEAR AIR CLEANING AND TREATMENT CONFERENCE}

\section{Computing the Efficiency of HEPA Filters Under DBA Conditions}

\section{General Procedure}

The determination of the efficiency of the HEPA filters under DBA conditions is a three step process.

(1) Determine the values of the temperature, moisture, radiation, and chemicals to which the final exhaust HEPA filters are exposed during normal operations and during and after the DBA. Standard engineering practices should be used in determining these parameter values. Also assume a filter age that represents the maximum age of filters in the facility. The most important parameter to be determined is the filter pressure drop during and after the DBA. Guides for estimating the pressure drop are presented in this paper.

(2) Compare the environmental and filter parameters listed in step (1) to the corresponding values in Table 5 that show the threshold value of differential pressures that would cause structural damage to the HEPA fitter. For multiple parameters, use the lowest differential pressure. Compute the differential pressure for the HEPA filter under the DBA conditions assuming maximum particle loading. If the filter differential pressure under DBA conditions is greater than the threshold value shown in Table 5 , then the HEPA filter is assumed to be damaged, and the allowed efficiency credit is $0 \%$.

(3) If the differential pressure is less than the threshold value shown in Table 5 , then the filter is considered to be undamaged, and the filter penetration is determined from the filter penetration values shown in Table 6 that correspond to the environmental coriditions in the DBA. For a DBA challenge with multiple environmental parameters, the total HEPA penetration is obtained by adding the penetrations from the individual environmental parameters.

\section{Examples}

Example 1: Assume a two-stage HEPA filter system with a demister system, a blower with a 15 inch w.g. vacuum and a DBA that consists of a hot fire followed by activation of a fire protection water deluge system to protect the first stage HEPA fitter. The temperature at the first filter is determined to be $250^{\circ} \mathrm{C}$ and the second $200^{\circ} \mathrm{C}$. In this scenario, the first filter would be partially loaded, but not completely plugged, due to the smoke. Using the data in Table 5, each filter has a maximum differential pressure threshold of 33 inches, beyond which value the filters will rupture. Since the fan can only pull 15 inches w.g., there is no damage to the filters at this point. However, once the water deluge is turned on, the first filter becomes wet and becomes plugged. The threshold value for filter rupture is now reduced to 10 
inches w.g. because the filter is wet. Since the blower vacuum exceeds the minimum value of differential pressure required to damage the filter, the first fitter will be structurally damaged. This follows because the full vacuum of the blower will be applied across the first stage HEPA filter. If the smoke from the fire and the water spray continues, then the second filter will soon plug and also rupture. The assumed efficiency for each ruptured filter is $0 \%$, thus resulting in $0 \%$ efficiency for the two filters.

Example 2: Assume the same conditions as in example 1, but the blower can only pull a 10 inch w.g. vacuum. In this case the threshold value for the differential pressure is still 33 inches w.g. for each of the two filters during the high temperature exposure. After the water spray is turned on, the differential pressure threshold for the first filter is 10 inches w.g. Since the fan can only pull 10 inches w.g., neither filter is damaged, but the first filter is plugged and the air flow is reduced to a very low flow. The first HEPA filter has a penetration of $1.4 \%$ according to Table 6 because $1.3 \%$ penetration due to moisture accumulation is computed from the liquid particle deposits and added to the baseline penetration of $0.1 \%$. Since the second HEPA filter has the baseline $0.1 \%$ penetration, the total penetration for the two filters is $0.0014 \%$, or an efficiency of $99.9986 \%$. However, the process air ventilation system is effectively shut down, and contaminants may leak out into the building rooms from the containment structure, ducts and housings.

Example 3: A two-stage HEPA filter that has a flow of $1,000 \mathrm{cfm}$ and an initial pressure drop of 4 inches w.g. for the first stage and 1 inch w.g. for the second stage is subjected to a negative 75 inch w.g. tornado condition. Assume that the calculations from the EVENT code [45] predict a pressure drop of 50 inches w.g. across both filters and a flow of $10,000 \mathrm{cfm}$. Under these conditions the first filter will have a pressure drop of 40 inches and the second filter 10 inches w.g. According to the data in Table 5, the first filter will exceed the threshold differential pressure of 37 inches w.g. and will be structurally damaged. After the first filter is destroyed, it will no longer impose a restriction on the air flow and a new flow computation is required. Assume that the new computations using EVENT show the remaining HEPA filter has a pressure drop of 25 inches w.g. and a flow rate of 15,000 cfm. Under these conditions, the second HEPA filter will remain undamaged. The damaged filter will be assigned $0 \%$ efficiency, and the efficiency of the second fitter determined from Table 6. Assuming the relationship between air flow and penetration in Table 6, we estimate that $15,000 \mathrm{cfm}$ air flow will result in a penetration of $4.2 \%$. After the tornado pulse has passed, the filter penetration will return to the baseline value $0.1 \%$ (99.9\% efficiency). Thus the efficiency of the HEPA fitter system is $95.8 \%$ during the tornado and $99.9 \%$ after. 


\section{3rd DOE/NRC NUCLEAR AIR CLEANING AND TREATMENT CONFERENCE}

\section{Vl. Conclusion}

We have established criteria for calculating the efficiency of HEPA filters in a DOE nonreactor nuclear facility during and after a Design Basis Accident (DBA). Previous efforts of selecting a single efficiency value for all accidents were unsatisfactory because the potential DBAs vary significantly among different facilities and operations and result in large variations in computed filter efficiencies, primarily due to the deterioration of the HEPA filter. The deterioration of the filter efficiency depends on the exposure parameters; in severe exposure conditions the filter will be structurally damaged and have a residual efficiency of $0 \%$. The large variation in filter performance makes it necessary to determine filter efficiency on a case-by-case basis. It also prevents the use of a limited number of tests to estimate the efficiency for a given DBA.

Despite a significant amount of data in the literature on the performance of HEPA filters under offnormal conditions, it is not sufficient to cover the full matrix of different values and combinations of the following parameters that weaken the filter or cause structural damage: age, moisture, chemical attack, high temperature, overpressure, seismic and rough handling, liquid and solid particle loading, and radiation. The primary reason for the large number of combinations of parameters is that the parameters are highly dependent and cannot be separated. Because of the large number of possible DBA conditions, the preferred method for determining the efficiency of HEPA filters under DBA conditions is to conduct experiments in a test stand that can simulate the DBA conditions. Unfortunately since this test stand is not available, a secondary method is required

The method for computing filter efficiencies that we selected was chosen to be consistent with the limited data available. We approximated the performance of HEPA filters under DBA conditions by separating the effect of filter structural damage from filter efficiency. In this fashion, we were able to significantly reduce the number of required test parameters to match the available literature data. Even with this approximation, there are large gaps and limitations in the data that introduce significant error in the estimates of HEPA filter efficiencies under DBA conditions. Because of this limitation, conservative values of filter efficiency were chosen when there was insufficient data.

The method we selected for estimating the efficiency of the HEPA filters under DBA conditions involves three steps. In the first step, the filter pressure drop and environmental parameters such as temperature and moisture are determined during and after the DBA.. The second step consists of comparing the filter pressure drop to a set of threshold values above which the filter is structurally damaged. If the filter pressure drop is greater than the threshold value, the filter is structurally damaged and is assigned $0 \%$ efficiency. If the pressure drop is less, then the fitter efficiency is determined in a third 
step where the efficiency is determined from literature data that matches the same exposure conditions. The efficiency of the HEPA filters within DOE facilities should be determined on a case-by-case basis.

\section{Acknowledgement}

This study was supported by the Department of Energy, Defense Programs, Office of Engineering and Operations Support.

\section{Bibliography}

1. American Society of Mechanical Engineers, "Nuclear Power Plant Air-Cleaning Units and Components", ASME Standard N509, 1989.

2. U.S. Department of Energy, "General Design Criteria Manual", DOE Order 6430.1A, April 6, 1989.

3. Scripsick, R.C., Smitherman, R.L., and McNabb, S.A. "Operational evaluation of the High Flow Alternative Filter Test System" Proceedings of 19th DOE/NRC Nuclear Air Cleaning Conference, CONF860820, National Technical Information Service, Springfield, VA 22161, pp 863-889, 1987.

4. Elder, J. ,Graf, J., Dewart, J., Buhl, T., Wenzel, W., Walker, L., and Stoker, A. "A guide to radiological accident considerations for siting and design of DOE nonreactor nuclear facilities" , LOS Alamos National Laboratory Report, RFP-3650, June 25, 1984.

5. Atomic Energy Commission, "HEPA Filtration Guidelines," AEC unpublished meeting notes, 1971.

6. Walker, E. "A summary of parameters affecting the release and transport of radioactive material from an unplanned incident" Bechtel Report BNFO-81-2, September, 1978.

7. Nuclear Regulatory Commission, "Design, testing, and maintenance criteria for post accident engineered-safety-feature atmospheric cleanup system air filtration and absorption units of light -watercooled nuclear power plants" NRC Regulatory Guide 1.52, Revision 2, March 1978. 


\section{3rd DOE/NRC NUCLEAR AIR CLEANING AND TREATMENT CONFERENCE}

8. American Society of Mechanical Engineers, "Testing of Nuclear Air Treatment Systems", ASME Standard N510, 1989

9. Burchsted, C.A.., Kahn, J.E., Fuller, A.B., "Nuclear Air Cleaning Handbook. Design, Construction, and Testing of High-Efficiency Air Cleaning Systems for Nuclear Applications," Energy Research and Development Administration, ERDA 76-21, Oak Ridge national Laboratory, 1976.

10. Rubow, K.L., and Liu, B.Y.H. "Characteristics of membrane filters for particle collection" Eluid Filtration: Gas. Volume L.ASTM STP 975, R.R. Raber, Ed., American Society for Testing and Materials, Philadelphia, 1986.

11. Bergman, W., Taylor, R.D., Miller, H.H., Biermann, A.H., Hebard, H.D., daRoza, R.A., and Lum, B.Y., "Enhanced filtration program at LLNL- a progress report," Proceedings of 15th DOE Nuclear Air Cleaning Conference, NTIS Springfield, VA, CONF-78819, pp. 1058-1099,1979.

12. Payet, S., Boulaud, D., Madelaine, G., and Renoux, A. "Penetration and pressure drop of a HEPA filter during loading with submicron liquid particles" J.Aerosol Sci., Vol 23 No. 7, pp 723-735, 1992.

13. Osaki, M. and Kanagawa, A. "Performance of high-efficiency particulate air filters under severe conditions", Nuclear Technology, Vol 85, p 274-284, 1989.

14. Ensinger, U., Ruedinger, V., and Wilhelm, J.G. "Efficiency of HEPA filters at elevated temperatures: investigations with the $\mathrm{TiO}_{2}$ test method" Proceedings of the 20th DOE/NRC Nuclear Air Cleaning Conference, CONF-880822, NTIS Springfield, VA, pp 334-348, 1989.

15. VanOsdell, D.W., Liu, B.Y.H., Rubow, K.L., and Pui, D.Y.H. "Experimental study of submicrometer and ultrafine particle penetration and pressure drop for high efficiency filters" Aerosol science and Technology, Vol 12, pp. 911-925, 1990.

16. Johnson, J., Beason, D., Smith, P., and Gregory, W., "The effect of age on the structural integrity of HEPA filters" Proceedings of the 20th DOE/NRC Nuclear Air Cleaning Conference, CONF-880822, pp 366-382, 1989.. 


\section{3rd DOE/NRC NUCLEAR AIR CLEANING AND TREATMENT CONFERENCE}

17 Bergman, W. and Biermann, A. "Effect of DOP heterodispersion on HEPA filter penetration measurements" Proceedings of 18th DOE Nuclear Airborne Waste Management and Air Cleaning Conference, NTIS Springfield VA, CONF-840806, pp 327-347, 1985.

18. International Atomic Energy Agency, "Particulate Filtration in Nuclear Facilities," Technical Reports Series No. 325, IAEA, Vienna, 1991.

19. Anderson, W.L. "Making sense of HEPA filtration" Filtration and Separation Nov/Dec, pp-439$442,1989$.

20. Tillery, M.I., "Determination of protection factors for tandem HEPA filters" Proceedings of the 27th Annual Meting of the Institute of Environmental Sciences, 1981.

21. Hetland, N. and Russell, J. "Adequacy of ventilation exhaust filtering system for new plutonium facilities" Proceedings of the 12th AEC Air Cleaning Conference, NTIS Springfield VA, CONF-720823, pp 619-637, 1973.

22. Frigerio, N. and Stowe, R. "Plutonium and uranium emission experience in U.S. nuclear facilities using HEPA filtration" in High Efficiency Aerosol Filtration in the Nuclear Industry, Seminar Proceedings, CEC, Luxembourg, pp 457-471, Feb. 1977.

23. Ortiz, J. "In-place testing of multiple stage filter systems without disruption of plant operations in the plutonium facility at Los Alamos", Proceedings of 18th DOE Nuclear Airborne Waste Management and Air Cleaning Conference", NTIS Springfield VA, CONF-840806, pp 299-310, 1985.

24. Gonzales, M., Elder,J., and Ettinger, H., "Performance of multiple HEPA filters against plutonium aerosol" Proceedings of 13th AEC Air Cleaning Conference, NTIS Springfield VA, CONF-740807, pp 501$525,1975$.

25. Gregory, W., Martin, R., Smith, P., and Fenton, D., "Response of HEPA filters to simulated accident conditions", Proceedings of the 17th DOE Nuclear Air Cleaning conference, NTIS Springfield VA, CONF--820833, pp.1051-1068, 1983.

26. Breschi, R., Cuccuru, A., and Lanza, S. 'Scanning electron microscopy of ULPA and HEPA filtering papers" Proceedings of the 20th DOE/NRC Nuclear Air Cleaning conference, CONF-880822, pp 304-324, 1989. 


\section{3rd DOE/NRC NUCLEAR AIR CLEANING AND TREATMENT CONFERENCE}

27. Ruedinger, V. and Wilhelm, J., "HEPA fitter response to high air flow velocities", Proceedings of the 17th DOE Nuclear Air Cleaning Conference, NTIS Springfield VA, CONF-820833, pp. 1069-1092, 1983.

28. Jones, L., "Effects of radiation on reactor confinement system materials", Proceedings of 12th AEC Air Cleaning Conference, NTIS Springfield VA, CONF-720823, pp.655-676, 1973.

29. Woodard, R., Grossaint, K., and McFeeters, T., "Exhaust filtration on gloveboxes used for aqueous processing of plutonium", Proceedings of 14th ERDA Air Cleaning Conference, NTIS Springfield VA, CONF-760822, pp 677-693, 1977.

30. Hamblin, C. and Goodchild, P.J. "The effects of temperature on HEPA media" Proceedings of the 22nd DOENRC Nuclear Air Cleaning Conference, NTIS Springfield VA, CONF-9020823, pp 327-335, 1993.

31. Pratt, R. and Green, B. "Performance testing of HEPA fitters under hot dynamic conditions", Proceedings of 18th DOE Nuclear Airborne Waste Management and Air Cleaning Conference, NTIS Springfield VA, CONF-840806, pp 1107-1127, 1985.

32. Pratt, R. "Performance of filters under hot dynamic conditions", in Gaseous Effluent Treatment in Nuclear Installations, G. Fraser and F. Luykx, Eds, Graham \& Trotman, pp 824-836, 1986.

33. Ruedinger,V., Ricketts, C., and Wilhelm, J., "Limits of HEPA-fitter application under high-humidity conditions", Proceedings of the 18th DOE Nuclear Airborne Waste Management and Air Cleaning Conference, NTIS Springfield VA, CONF-840806, pp 1058-1084, 1985.

34. Ricketts, C. Ruedinger, V., and Wilhelm, J. "HEPA -filter behavior under high humidity airflows" in Proceedings of the 19th DOE/NRC Nuclear Air Cleaning Conference, NTIS Springfield VA, CONF$860820, p p 319-352,1987$

35. Brasseli, G.W. and Thorvaldson, W.G. "Development and evaluation of acid-resistant HEPA fitter media" in Proceedings of 17th DOE Nuclear Air Cleaning Conference, NTIS Springfield VA, CONF820833, pp 1125-1133., 1983.

36. Hackney, S. "Fire testing of HEPA filters installed in filter housings" in Proceedings of the 17th DOE Nuclear Air Cleaning Conference, NTIS Springfield VA, CONF-820833, pp 1030-1050, 1983. 


\section{3rd DOE/NRC NUCLEAR AIR CLEANING AND TREATMENT CONFERENCE}

37. First, M. "Performance of absolute filters at temperatures from ambient to $1000 \mathrm{~F}$ " in Proceedings of 12th AEC Air Cleaning Conference, NTIS Springfield VA, CONF-720823, pp 677-702, 1973.

38. Bergman, W., Elliott, J., and Wilson, K., "Performance of HEPA filters at LLNL following the 1980 and 1989 earthquakes" in Proceedings of the 23rd DOE/NRC Nuclear Air Cleaning and Treatment Conference, 1995.

39. U.S. Army, "Filter, particulate, high-efficiency, fire resistant," Military Specification MIL-F-51068, Commander, U.S. Army Armament, Munitions and Chemical command, ATTN; DRSMC-CLWEJA, Aberdeen Proving Ground, MD 21010-5423., August 11, 1988

40. Smith, P., Leslie, I.H., Hensel, E.C., Schultheis, T.M., and Walls, J.R., "Structural testing of salt loaded HEPA filters for WIPP" Proceedings of 22nd DOE/NRC Nuclear Air Cleaning Conf., NTIS Springfield VA, CONF-9020823, pp 262-280, 1993.

41. Gilbert, H., Fretthold, J.K., Rainer, F., Bergman, W., and Beason, D. "Preliminary studies to determine the shelf life of HEPA filters " in Proceedings of 23rd DOE/NRC Nuclear Air Cleaning and Treatment Conference,1995.

42. Alvares, N., Beason, D., Bergman, W., Ford, H., and Lipska, A. "Fire protection countermeasures for containment ventilation systems" in Proceedings of 16th DOE Nuclear Air Cleaning Conference, NTIS Springtield VA, CONF-801038, pp 1213-1277, 1981.

43. First, M.W., and Price, J.M. "Performance of 1000- and $1800 \mathrm{cfm}$ HEPA filters on long exposure to low atmospheric dust loadings, III" in Proceedings of 17th DOE Nuclear Air Cleaning Conference, NTIS Springfield VA, CONF-820833, pp909-918, 1983.

44. Novick, V.J., Klassen, J.F., and Monson, P.R., "Predicting mass loading as a function of pressure difference across prefilter/HEPA filter system, in Proceedings of the 22nd DOENRC Nuclear Air Cleaning Conference, NTIS Springfield VA, CONF-9020823, PP 554-573, 1993.

45 Gregory, W., Andrae, R., Duerre, K., Horak, H., and Smith, P., "HEPA filter response to simulated tornado loadings" in Proceedings of 15th DOE Nuclear Air Cleaning Conference, NTIS Springfield VA, CONF-780819, pp 694-706., 1979. 